\title{
Mars Dust Storm Effects in the Ionosphere and Magnetosphere and Implications for Atmospheric Carbon Loss
}

\author{
Xiaohua Fang ${ }^{1}$, Yingjuan $\mathrm{Ma}^{2}$, Yuni Lee ${ }^{3}$, Stephen Bougher ${ }^{4}$, Guiping Liu ${ }^{5}$, Mehdi Benna \\ ${ }^{3}$, Paul Mahaffy ${ }^{3}$, Luca Montabone ${ }^{6,7}$, David Pawlowski ${ }^{8}$, Chuanfei Dong ${ }^{9}$, Yaxue Dong ${ }^{1}$, \\ and Bruce Jakosky ${ }^{1}$ \\ ${ }^{1}$ Laboratory for Atmospheric and Space Physics, University of Colorado, Boulder, Colorado, USA \\ ${ }^{2}$ Department of Earth, Planetary and Space Sciences, University of California, Los Angeles, California, USA \\ ${ }^{3}$ NASA Goddard Space Flight Center, Greenbelt, Maryland, USA \\ ${ }^{4}$ Department of Climate and Space Sciences and Engineering, University of Michigan, Ann Arbor, Michigan, USA \\ ${ }^{5}$ Space Sciences Laboratory, University of California, Berkeley, California, USA \\ ${ }^{6}$ Space Science Institute, Boulder, Colorado, USA \\ ${ }^{7}$ Laboratoire de Météorologie Dynamique/IPSL, Sorbonne Université, Paris, France \\ ${ }^{8}$ Physics and Astronomy Department, Eastern Michigan University, Ypsilanti, Michigan, USA \\ ${ }^{9}$ Department of Astrophysical Sciences and Princeton Plasma Physics Laboratory, Princeton University, Princeton, New \\ Jersey, USA
}

\begin{abstract}
Key Points:
- The dayside main ionosphere is lifted in accordance with dust-induced atmospheric expansion, with peak electron densities unchanged.

- Dust-induced perturbations propagate upward from the ionosphere to the magnetosphere and extend from the dayside to the nightside.

- Strong dust storms may enhance $\mathrm{CO}_{2}^{+}$loss by a factor of $\sim 3$ and increase total carbon loss (neutrals and ions) by $\sim 20 \%$ or more.
\end{abstract}

This is the author manuscript accepted for publication and has undergone full peer review but has not been through the copyediting, typesetting, pagination and proofreading process, which may lead to differences between this version and the Version of Record. Please cite this article as doi: 10.1029/2019JA026838

Corresponding author: Xiaohua Fang, Xiaohua.Fang@lasp.colorado.edu

This article is protected by copyright. All rights reserved. 


\begin{abstract}
Mars regional and global dust storms are able to impact the lower/upper atmospheres through dust aerosol radiative heating and cooling and atmospheric circulation. Here we present the first attempt to globally investigate how the dust impact transfers from the neutral upper atmosphere to the ionosphere and the induced magnetosphere above $100 \mathrm{~km}$ altitude. This is achieved by running a multifluid magnetohydrodynamic model under nondusty and dusty atmospheric conditions for the 2017 late-winter regional storm and the 1971-1972 global storm. Our results show that the dayside main ionospheric layer (below $\sim 250 \mathrm{~km}$ altitude) undergoes an overall upwelling, where photochemical reactions dominate. The peak electron density remains unchanged, and the peak altitude shift is in accordance with the upper atmospheric expansion $(\sim 5 \mathrm{~km}$ and $\sim 15 \mathrm{~km}$ for the regional and global storms, respectively). Controlled by the day-to-night transport, the nightside ionosphere responds to the dust storms in a close connection with what happens on the dayside but not apparently with the ambient atmospheric change. At higher altitudes, dustinduced perturbations propagate upward from the ionosphere to the magnetosphere and extend from the dayside to the nightside, within a broad region bounded by the induced magnetospheric boundary. It is found that the global dust storm is able to dramatically enhance the $\mathrm{CO}_{2}^{+}$loss by a factor of $\sim 3$, which amounts to an increase of $\sim 20 \%$ or more for total carbon loss (in the forms of neutrals and ions). Strong dust storms are a potentially important factor in atmospheric evolution at Mars.
\end{abstract}

\title{
1 Introduction
}

Today's Mars is a dry and dusty planet, on which dust storms frequently occur mainly during southern hemisphere spring and summer seasons [e.g., Zurek, 1982]. When dust storms happen, a significant amount of dust particles are injected into the atmosphere by wind-related processes. The most common are local dust storms that have limited occurrence scale (size and duration) and relatively low intensity and extent of dust opacity. Sometimes local storms merge and develop into a continent-sized, regional dust storm, which may last for weeks or more. Beyond local and regional storms, Mars has some of the greatest dust storms in the solar system, which occur infrequently but are able to obscure the planet's surface and last for several months. This type of planet-encircling dust storm is called a great/global dust storm or a global dust event, and receives much atten- 
tion from the scientific community and the public. More detailed discussions on local, regional, and global dust storms have been given by, e.g., Cantor et al. [2001].

Mars dust storms are well known to cause perturbations in the neutral temperature, wind, and density of the lower and even upper atmosphere [e.g., Haberle et al., 1982; Zurek, 1992; Bougher et al., 1997; Keating et al., 1998; Bougher et al., 1999; Smith et al., 2002; Forget et al., 2009; Medvedev et al., 2013; Withers and Pratt, 2013; Bougher et al., 2017; Kahre et al., 2017; Toigo et al, 2018; Liu et al., 2018; Montabone and Forget, 2018]. The dust-induced atmospheric disturbance is associated with a series of complex direct and indirect processes: initially involved with dust aerosol radiative heating and cooling, followed by significant alteration of the atmospheric thermal structure and circulation. These dust-related radiative and dynamical processes result in profound atmospheric changes, which may be roughly regarded as an expansion of the entire atmospheric column particularly at high altitudes [e.g., Kliore et al., 1973; Withers and Pratt, 2013]. It is recognized that dust storms play an important role in governing the atmosphere and climate of Mars. It is worth noting that although dust particles may be carried by vertical transport to altitudes as high as $80 \mathrm{~km}$ [Clancy et al., 2010], there is no evidence supporting that direct dust loading would happen higher. The dust impact in the upper atmosphere $(>80 \mathrm{~km})$ is basically an indirect effect resulting from the coupling with the lower atmosphere [Bougher et al., 1997].

Unlike extensive studies on the neutral atmospheric effectiveness of dust storms, their impact on the charged particle radiation environment near Mars remains poorly understood. Kliore et al. [1973], Hantsch and Bauer [1990], and Zhang et al. [1990] analyzed radio occultation measurements by the Mariner 9 spacecraft and reported that the main ionospheric layer of Mars (which typically peaks at $\sim 120 \mathrm{~km}$ altitude or higher, owing to the absorption of the solar extreme ultraviolet, EUV, radiation) behaved differently during the 1971-1972 global dust storm. They reported that the ionospheric peak altitude was considerably upward lifted by $\sim 20 \mathrm{~km}$, although the peak electron density was normal and in line with Chapman theory predictions. Wang and Nielsen [2003] used a 1-D photochemical model to simulate the ionospheric response during this specific dust storm. Their results showed that the ionospheric altitude profile underwent an overall upward lift, maintaining the similar peak intensity at a significantly elevated altitude. The numerical work of Wang and Nielsen [2003], consistent with the Mariner 9 data interpretation, implies that it is the expanded upper neutral atmosphere that is responsible for the ionospheric 
anomaly during the dust storm, a process controlled by the altitude-varying energy deposition by the solar EUV irradiance (i.e., photoionization) and subsequent photochemical reactions. In addition, a handful of studies have been reported on the dust influence in the regions other than the main ionospheric layer. Haider et al. [2010] and Nemec et al. [2015] found that ion/electron concentrations at low altitudes $(<60 \mathrm{~km})$ may be significantly depleted during dust storms due to the reduction of ionizing galactic cosmic rays as a result of the enhanced optical depth. Liemohn et al. [2012] analyzed dayside photoelectron flux observations of the Mars Global Surveyor (MGS, at 400 km altitude), and found that statistically significant correlations were achieved with the dust-modulated solar EUV intensity after taking into account the dust opacity over the preceding 7-month time history. The work speculates that the long-lived dust influence on photoelectrons may be attributed to the composition/density change of the upper atmosphere, which is also supported by $X u$ et al. [2014].

Despite these early efforts, there is still no global picture of how and the extent to which the entire Mars plasma environment (including the ionosphere and the induced magnetosphere) reacts to dust storms that develop and arise from the surface. Besides what we have learned from those sparse spatial and temporal sampling, what other disturbances are there during dust storms? In particular, little is known about how high in altitude dust storms are capable of extending their impact beyond the low-altitude part of the ionosphere. It is natural to expect that upper atmospheric density changes in dust storms would result in photoionization rate perturbations and then have an impact on the ionosphere and ultimately manifest themselves at higher altitudes through the interaction of the Mars conductive obstacle with the solar wind. The main difficulty, however, is on a quantitative assessment of how important these potential consequences are and whether they may be distinguishable from other sources of variability. In contrast with the highly collisional bottomside ionosphere (which is in relatively closer proximity to dust activity regions), the plasma distribution at high altitudes is in a collisionless regime and is dominated by transport processes, that is, is more dynamic in nature. The continuous change of observational sites and conditions in reality, together with the lack of simultaneous multipoint measurements, constitute a practical challenge of organizing data and extracting the effects that may be reliably associated with dust activity.

Our current strategy to overcome the limitations in observational data analysis is to apply a state-of-the-art global magnetohydrodynamic (MHD) model [Najib et al., 2011; 
Dong et al., 2018a] using physically realistic background and boundary conditions for specific dust storm events. Numerical simulations are conducted under observationally constrained atmospheric conditions, separately corresponding to nondusty and dusty scenarios, with all the other model parameters held unchanged. By making direct comparison of the results between these controlled runs, this study represents the first attempt to assess the ionospheric and magnetospheric disturbances on a planetary scale during dust storms and to discuss the implications for total atmospheric loss.

\section{The Global and Regional Dust Storms for Case Studies}

Figure 1 gives an overview of the zonally-averaged dust opacity in infrared over 11 Martian years (MYs, from MY 24 to currently MY 34), as a function of the solar longitude $\left(L_{S}\right)$ and planetary latitude. The column dust optical depth (CDOD) is derived using combined infrared radiance observations from several Mars orbiters: the Thermal Emission Spectrometer (TES) onboard Mars Global Surveyor [Christensen et al., 2001], the Thermal Emission Imaging System (THEMIS) onboard Mars Odyssey [Christensen et al., 2004], and the Mars Climate Sounder (MCS) onboard Mars Reconnaissance Orbiter [McCleese et al., 2004]. The algorithms for synthesizing these measurements to derive dust opacity products have been described in detail by Montabone et al. [2015] and are not repeated here. Note that Figure 1 is a replot of Figure 16 of Montabone et al. [2015] for MYs 24-31, updated with latest results for MYs 32 and 33 using the same data processing technique. MY 34 is produced using specific processing described in detail by Montabone et al. [2019] within this special issue. Due to the differences in data processing techniques (see the Appendix of Montabone et al. [2019] for further details), particular caution is required for a direct comparison of the zonal means between MY 34 and other Martian years (particularly MYs 28-33).

The time series of the dust opacity presented in Figure 1 clearly demonstrates that Mars atmospheric dust loading in general follows an annual repeatable pattern with a strong seasonal dependence. A Martian year can be roughly divided into two seasons in terms of atmospheric dust loading [e.g., Montabone and Forget, 2018]. A "low dust loading" season starts sometimes after the northern hemisphere vernal equinox $\left(L_{S} \sim 10\right)$ and ends sometimes before the autumnal equinox $\left(L_{s} \sim 140\right)$. Mars generally is calm within this season except for local dust storms and dust devils. During the rest of the time, Mars is inside a "high dust loading" season, when the planet receives intensified solar heating 
particularly near its perihelion $\left(L_{s}=251\right)$. Regional dust storms start to happen within the dust season. Outside annually repeatable patterns of dust opacity, planet-encircling or global dust storms are episodic, powerful meteorological phenomena, which lift a considerable amount of dust particles into the atmosphere and obscure most of the planet's surface. Their exact timing of occurrence is (so far) unpredictable, on an intermittent basis of roughly every two to three Martian years [e.g., Zurek and Martin, 1993]. Three global dust storms are visible in Figure 1, showing distinct optical depth enhancement over nearly all latitudes in years 2001 (MY 25), 2007 (MY 28), and 2018 (MY 34), respectively. See Montabone and Forget [2018] and references therein for more discussions of the two-dustseason partition and more specificity of global dust events. Figure 2 gives a 3-D view of CDOD global distributions at an $L_{s}$ cadence of $30^{\circ}$ during MY 34. The overall seasonal evolution of dust loading during this specific MY is consistent with other MYs (Figure 1). The 2018 planet-encircling dust storm is readily seen in Figure 2 in the dramatic dust optical depth increase, in both magnitude and spatial extent, particularly at $L_{S}=210^{\circ}$ and $240^{\circ}$.

In this study, we select one relatively weak regional storm and one strong global storm for numerical simulation. The comparison of the Mars ionospheric and magnetospheric responses to different storm levels enables an assessment of the range of potential dust consequences. Considering that it is the plasma regime rather than the neutral atmospheric regime that our current dust impact study focuses on, we rely on previously published works on atmospheric changes from nondusty to dusty scenarios, which serve as direct inputs to our MHD model. For a regional storm, we select the one in year 2017 (hereinafter referred to as event 1), highlighted by the red box in Figure 1. The upper atmospheric conditions within the regional storm have been available, which were reported by Liu et al. [2018] using the in-situ measurements from the NASA Mars Atmosphere and Volatile EvolutioN (MAVEN) mission [Jakosky et al., 2015]. On the other hand, an ideal global storm case for study would be the recent one in year 2018 (MY 34), which receives the most comprehensive measurements and is the focus of the current special issue. Unfortunately, the evaluation of the dust atmospheric impact for the 2018 global storm is still an ongoing process and the results are not yet publicly available. As an alternative, we select the 1971-1972 global dust storm (hereinafter referred to as event 2), whose atmospheric changes have been estimated by Wang and Nielsen [2003] and are available for direct use. By limiting our case studies to those storms whose neutral atmospheric estimates are al- 
ready available, the current work is able to maintain a focused scope on studying the dust impact on the charged particle regime.

\section{Multifluid MHD Model of Mars}

\subsection{Model Description}

The primary research tool for this work is the 3-D multifluid MHD model of $\mathrm{Na}$ jib et al. [2011] and Dong et al. [2018a,b]. After two decades of code development and improvement since Liu et al. [1999] and Ma et al. [2004], the MHD model provides a state-of-the-art solution of the interaction between the incoming solar wind and the Mars conductive obstacle (consisting of the ionosphere and planet-attached crustal magnetic anomalies). The MHD continuity, momentum, and pressure equations are solved separately for each of the major ion species $\left(\mathrm{H}^{+}, \mathrm{O}_{2}^{+}, \mathrm{O}^{+}, \mathrm{CO}_{2}^{+}\right)$under the multifluid approximation. The sources for planetary ions include photoionization of a prescribed global atmosphere $\left(\mathrm{CO}_{2}, \mathrm{O}, \mathrm{H}\right)$, charge exchange collisions, and electron impact ionizations. The effect of the neutral wind is currently neglected in the MHD model. The magnetic field is self-consistently calculated with the plasma distribution, dominated by the crustal magnetic field on the inner model boundary of $100 \mathrm{~km}$ altitude and by the interplanetary magnetic field (IMF) in the upstream. The MHD equations are solved in the classic Marscentered Solar Orbital (MSO) coordinate system. The simulation domain is sufficiently broad to allow for the interaction of the plasma and fields of the solar and planetary origins: $-36 R_{M} \leq X \leq 12 R_{M},-24 R_{M} \leq Y, Z \leq 24 R_{M}$, where $R_{M}$ stands for the mean Martian radius of $3396 \mathrm{~km}$. To ensure adequate sensitivity for the ionospheric response to upper atmospheric changes, we adopt the highest spatial resolutions that have ever been realized in the MHD model: $1.5^{\circ}$ in both longitudinal and latitudinal directions and an altitude resolution of $2.5 \mathrm{~km}$ in the ionosphere. At altitudes higher than $\sim 1000 \mathrm{~km}$, the angular resolution is relaxed to $3^{\circ}$, and the radial resolution doubles and then gradually increases with altitude as in typical MHD runs.

Our current investigation focuses on spatial rather than temporal variability of the dust impact. Accordingly, the background conditions for our model runs are set to be static, or time independent. This is not possible in reality but is a particular advantage of numerical experiments. This is also a strategy commonly used by the global Mars-solar wind interaction modeling community, not mentioning that running the multifluid MHD 
model in a time-varying fashion is prohibitive at present in terms of computation time. The nominal solar wind has a number density of $n_{s w}=4 \mathrm{~cm}^{-3}$ and an antisunward flow speed of $U_{s w}=400 \mathrm{~km} / \mathrm{s}$, corresponding to a dynamic pressure of $1.07 \mathrm{nPa}$. The IMF is a Parker spiral of $\left(B_{X}, B_{Y}, B_{Z}\right)=(-1.634,2.516,0) \mathrm{nT}$, making the MSO coordinate system for MHD calculation identical to the Mars-Solar-Electric field (MSE) coordinate system. The planetary axis is tilted in the MSO direction of $(-0.224,0.362,0.905)$ and $(-0.412$, $0.106,0.905$ ) for event 1 and event 2 , respectively, using representative time points of 2017-03-06 and 1971-11-01. The subsolar longitude, and thus the planet's orientation to the Sun, are specified in such a way that the strongest crustal magnetic field region (near $178^{\circ} \mathrm{E}, 53^{\circ} \mathrm{S}$ ) is located on the nightside with the maximum solar zenith angle (SZA). This ensures that the potentially important crustal field influence on plasma distributions is minimized [cf., e.g., Ma et al., 2014; Fang et al., 2015, 2017]. In addition, ionizing solar irradiance is parameterized by $10.7-\mathrm{cm}$ radio fluxes at the Mars' orbit, which are estimated by scaling Earth observations with inverse square of the Sun-Mars distance in AU. The Mars equivalent $F_{10.7}$ values are 32.6 (in event 1) and 54.9 (in event 2), which are used to interpolate $\mathrm{CO}_{2}$ and $\mathrm{O}$ photoionization frequencies according to Table 9.2 of Schunk and Nagy [2009]. Note that the MHD model in its current form adopts $F_{10.7}$-parameterized photoionization frequencies, and thus cannot take advantage of the detailed solar flux measurements from MAVEN EUV Monitor [Eparvier et al., 2015].

\subsection{Atmospheric Conditions for Model Input}

MHD calculations are performed twice for each of the two dust events to be studied, under nondusty and dusty atmospheric conditions, respectively. For the two controlled runs of each event, the parameters other than neutral species distributions are specified to be the same to exclude unnecessary interference and thus to effectively concentrate on dust effects. Note that all the four atmospheric conditions (two events by two nondusty-dusty settings) need to be specifically characterized, given the fact that the timing (i.e., seasonal effects) and solar irradiance of the events are different. There is no need for considering dust aerosols in our MHD simulations, because direct dust effects are negligible beyond $100 \mathrm{~km}$ altitude (which is the location of the bottom boundary of our model). At high altitudes, dust storms act in such an indirect way that the dust-induced perturbations in neutral species distributions impact the plasma regime via ion-involved chemical reactions and ion-neutral momentum/energy transfer collisions. Since neutral concentrations are many 
orders of magnitude greater than those of charged particles, the atmospheric distribution serves as a static input to the model. Without any specific knowledge of the 3-D atmospheric distributions, we make an assumption of a horizontally uniform spherically symmetric atmosphere. This is a reasonable first-order approximation, given that dust storm effects in the upper atmosphere are found to have much broader horizontal scales [Withers and Pratt, 2013].

Figure 3 shows the specification of the nondusty and dusty atmospheric conditions for event 1 . The circles indicate estimates from the MAVEN instrument of the Neutral Gas and Ion Mass Spectrometer (NGIMS) [Mahaffy et al., 2014]. The dusty $\mathrm{CO}_{2}$ and $\mathrm{O}$ densities (red and dark blue circles, respectively) are the average over 15 MAVEN orbits ( $\sim 3$ days) near $L_{s}=328$, which corresponds to the third dust increase episode in Liu et al. [2018] and is marked by dashed line in Figure 1. For nondusty conditions, we choose not to use the average levels of pre-storm densities. The study of Liu et al. [2018] demonstrates that due to the continuous change of the MAVEN periapsis segments in longitude, latitude, local time, and SZA, the density contrast between the storm and pre-storm local values is not necessarily primarily caused by dust activity. Therefore we take a conservative approach for estimating the nondusty atmosphere by using the 25th percentile within the $L_{s}$ range of 310-360, as denoted by the red box in Figure 1 .

Next we fit and extrapolate these MAVEN data products into both lower and higher altitudes to fill in the MHD simulation domain under a globally symmetric approximation. A multi-component approximation is adopted to fit the MAVEN data, with various atmospheric thermal and hot components considered as follows. A $m$-component structure $\left(m=2 / 4 / 2\right.$ for $\mathrm{CO}_{2} / \mathrm{O} / \mathrm{H}$, respectively) is defined as $n(h)=\sum_{i=1}^{m} n_{0 i} \cdot \exp \left(-(h-100) / H_{i}\right)$, where $n$ is the total number density of an atmospheric species $\left(\mathrm{CO}_{2}, \mathrm{O}, \mathrm{H}\right)$ at altitude $h$. On the right hand side of the equation, $n_{0 i}$ and $H_{i}$ stand for the density at $h=100 \mathrm{~km}$ and the scale height in units of $\mathrm{km}$ for the $i$ th $(i=1, \ldots, m)$ component. The nominal, solar minimum atmosphere that is typically adopted by the MHD model is superposed in Figure 3 for reference, with dashed lines for $\mathrm{CO}_{2} / \mathrm{O}$ and a green line for $\mathrm{H}$ [Ma et al., 2004]. Recall that the average solar EUV levels during both dust events are much closer to solar minimum than to solar maximum (see section 3.1). For simplicity, this study directly takes the reference $\mathrm{H}$ profile for nondusty and dusty conditions at all altitudes for two considerations. Firstly, the proton contribution from planetary hydrogen is dominated by that of solar wind origin at high altitudes [Najib et al., 2011]. Secondly, at low altitudes, iono- 
spheric $\mathrm{H}^{+}$is a minor species in comparison with abundant $\mathrm{O}_{2}^{+}$and $\mathrm{CO}_{2}^{+}$. Therefore, no important errors would be expected from the simplification on the planetary $\mathrm{H}$ distribution. Below the MAVEN periapsis altitude where no specifics are available for $\mathrm{CO}_{2}$ and $\mathrm{O}$, we assume that their minimum scale heights in the $m$-component approximation (that is, for the dominant components near $100 \mathrm{~km}$ ) approach those of the reference atmosphere. To self consistently describe the oxygen corona above $\sim 250 \mathrm{~km}$ altitude, we take an iterative approach. In the first iteration run, we assume the hot $\mathrm{O}$ components from the reference atmosphere and make multi-component $\mathrm{O}$ and $\mathrm{CO}_{2}$ atmospheric fits on a least-squares scheme over the entire altitude range $(\geq 100 \mathrm{~km})$. We use these estimated atmospheric profiles to perform an exploration MHD run and obtain ionospheric properties. The Mars Adaptive Mesh Particle Simulator (AMPS) of Lee et al. [2015a, 2019] is applied to account in detail for the dissociative recombination of ionospheric $\mathrm{O}_{2}^{+}\left(\mathrm{O}_{2}^{+}+\mathrm{e}^{-} \rightarrow \mathrm{O}\right.$ $+\mathrm{O})$ and thus energetic $\mathrm{O}$ production and collisions with ambient neutrals. The AMPScalculated, high-altitude oxygen distributions are seen in Figure 3c. We then perform the second iteration run, which is similar to the first one except that the AMPS results rather than the reference $\mathrm{O}$ corona are used for atmospheric fits. The final atmospheric $\mathrm{CO}_{2}$ and O specifications are presented in solid lines in Figure 3, which serve as background input conditions for the MHD model. More detailed discussions of the AMPS-calculated hot oxygen corona for these specific cases have been given by Lee et al. [2019].

The above procedures are followed separately to derive both nondusty and dusty atmospheric profiles. Below the altitude of MAVEN data availability, we make an ad-hoc adjustment for the dusty estimation. It is assumed that for both $\mathrm{CO}_{2}$ and $\mathrm{O}$, the nondustyto-dusty density enhancement factors at $\sim 160 \mathrm{~km}$ are extended unchanged down to the lower boundary of the model (i.e., $100 \mathrm{~km}$ ). Using the scale heights of the density distributions, we calculate neutral temperatures of each species and then thermal pressures. Figure $3 \mathrm{~b}$ shows the atmospheric pressure and bulk temperature results under nondusty and dusty conditions in event 1 . It is seen that the entire upper atmosphere is approximately lifted by $>5 \mathrm{~km}$ in altitude in response to this regional storm, and there is no important change in the bulk temperature. The low-altitude temperature overlap is caused by the aforementioned ad-hoc approximation. A more careful examination is needed for the validity of the temperature difference above $\sim 160 \mathrm{~km}$ altitude, given the uncertainties due to our NGIMS data averaging (which is independently performed at altitude levels) and the simplistic fitting. Nevertheless, our numerical experiments showed that the MHD 
results are not sensitive to the neutral temperature setup. It is also found by Wang and Nielsen [2003] that the neutral temperature makes an insignificant impact on the ionospheric density. Although the atmospheric expansion (in total pressure/density) effectively stops at $\sim 300 \mathrm{~km}$ altitude (Figure 3d), significant compositional changes (specifically in the $\mathrm{O}$ corona) take place at higher altitudes up to $\sim 500 \mathrm{~km}$ (Figure $3 \mathrm{c}$ ).

A similar approach is applied to specify atmospheric conditions during the 19711972 global storm, except that the density estimates between $100-180 \mathrm{~km}$ are directly adopted from Figure 3 of Wang and Nielsen [2003]. Our estimation results over the MHD spatial domain are presented in Figure 4. Several differences in the upper atmospheric responses between the regional and global dust storms are noticeable in the comparison of Figure 4 to Figure 3. The atmosphere expands more significantly in event 2 showing an upwelling of 10-15 km in altitude (Figure 4b), which is expected owing to enhanced lower atmospheric dust load and solar radiation absorption. The neutral temperature change, which decreases with altitude, indicates that direct dust aerosol heating happens mostly in the lower atmosphere. In addition, an important density drop of about $35 \%$ happens in the O corona, nearly altitude independent above $\sim 650 \mathrm{~km}$ (Figure $4 \mathrm{c}$ ), which is mainly due to the enhanced collisional loss of energetic $\mathrm{O}$ in the thicker $\mathrm{CO}_{2}$ atmosphere [Lee et al., 2018, 2019].

\section{Multifluid MHD Results}

\subsection{Dust Impact on the Ionosphere and Magnetosphere}

We conduct multifluid MHD simulations using the estimated boundary conditions (in solar radiation, solar wind, IMF, crustal magnetic anomalies) and background atmospheric conditions, which are described in section 3. The model is run sufficiently long until a dynamic equilibrium is achieved in the Mars system. A total of 4 steady-state model runs have been obtained in correspondence with 2 nondusty/dusty atmospheric settings for 2 dust events. Figure 5 and Figure 6 show the comparison of the ionospheric properties at the subsolar point $\left(\mathrm{SZA}=0^{\circ}\right)$ between nondusty and dusty conditions for the regional and global dust storms, respectively. One prominent feature in both Figures 5a and $6 \mathrm{a}$ is that the main ionosphere may be roughly seen as being subject to an upward lift in response to dust-induced atmospheric expansion. The charged particle density peaks are located at significantly higher altitudes during the dusty scenarios, with their peak 
intensities barely affected (except for $\mathrm{O}^{+}$in event 2 ). This result is consistent in quality with the report of Wang and Nielsen [2003], which used a simplified 1-D photochemical ionospheric model. Our MHD results show a subsolar peak altitude lift of approximately $5 / 5 / 5 / 15 \mathrm{~km}$ and $15 / 12.5 / 17.5 / 17.5 \mathrm{~km}$ for $\mathrm{e}^{-} / \mathrm{O}_{2}^{+} / \mathrm{CO}_{2}^{+} / \mathrm{O}^{+}$in event 1 and in event 2 , respectively. As a consequence, density increases (decreases) occur at altitudes above (below) their peak locations.

A close look at Figure 5b reveals that over a broad altitude range in event 1, the two major ionospheric ions of $\mathrm{O}_{2}^{+}$(in blue) and $\mathrm{CO}_{2}^{+}$(in red) share a similar percentage density change of about $30 \%-40 \%$ with $\mathrm{e}^{-}$(or total ions, in black); in other words, the mixing ratios and thus the ionospheric composition of the major ions are insignificantly affected during the regional dust storm. To test the hypothesis that the ionosphere seems to exhibit a simple upwelling in event 1 , the results are reorganized according to atmospheric thermal pressure levels (in place of altitude levels) and are presented in Figures $5 \mathrm{c}$ and $5 \mathrm{~d}$. As speculated, the ionospheric profiles become nearly identical with respect to the neutral pressure. Figure 5d illustrates that in the main ionosphere (corresponding to neutral pressure higher than $10^{-8} \mathrm{~Pa}$, or at altitudes lower than $220 \mathrm{~km}$, see Figure $3 \mathrm{~b}$ ), almost all charged particles have a negligible density change within $\pm 10 \%$. Noticeable exceptions happen at higher altitudes, particularly for $\mathrm{O}^{+}$(in brown). Even in terms of neutral pressure as vertical coordinate, the $\mathrm{O}^{+}$density shows an increase by up to a factor of 3, which, nevertheless, is considerably smaller than that in the altitude cooridinate as seen in Figure 5b. The different responses of $\mathrm{O}^{+}$than the heavier ions to the atmospheric lift are understandable. The ion production of $\mathrm{CO}_{2}^{+} / \mathrm{O}_{2}^{+}$is concentrated at low altitudes, which originates from the solar EUV absorption by heavy atmospheric $\mathrm{CO}_{2}$ molecules. Photoionization rates are directly determined by local quantities of neutral densities and ionizing solar fluxes, the latter of which are controlled by the optical depth. Being a proxy of the overlying atmospheric column mass, the neutral pressure regulates the optical depth of solar radiation and is thus (nonlinearly) correlated with ion production. Given that the primary $\mathrm{CO}_{2}^{+} / \mathrm{O}_{2}^{+}$production is within a photochemical equilibrium region, it is not surprising to see that these heavier ions vertically shift their density profiles in accordance with the moderate shift of the neutral pressure. The vertical distribution of $\mathrm{O}^{+}$, however, is much more complex, which peaks at high altitudes where vertical transport starts to dominate over the photochemical process. Because the $\mathrm{O}^{+}$production itself peaks at low altitudes [e.g., Fox, 2004], the dust-induced vertical shift of the atmospheric pressure does 
indeed affect the location of $\mathrm{O}^{+}$production but cannot solely account for the distribution of the ion density. This is the reason why we see the smaller but still significant change for $\mathrm{O}^{+}$in Figure 5d than in Figure 5b. It should be pointed out that the great percentage differences for $\mathrm{CO}_{2}^{+} / \mathrm{O}_{2}^{+}$densities at the top of Figure $5 \mathrm{~d}$ (with $<1 \mathrm{nPa}$ pressures, or $>600$ $\mathrm{km}$ altitudes) are not that meaningful for two reasons. First, their abundances are dominated by the lighter species like $\mathrm{H}^{+}$and $\mathrm{O}^{+}$. Second, global transport is so important at high altitudes as to require a careful evaluation over a broad spatial scale instead of being limited to along the radial direction as examined here.

Figure 6 shows the results of event 2 using a similar format as Figure 5. Several distinct differences stand out during the global dust storm. First, while the peak locations of ionospheric ions are also lifted in consistence with the upper atmospheric expansion, the concentration of $\mathrm{O}^{+}$significantly decreases at all altitudes: up to about $-80 \%$ near 210 $\mathrm{km}$. This is mainly caused by the enhanced ion loss through charge exchange collisions with ambient $\mathrm{CO}_{2}$ neutrals $\left(\mathrm{O}^{+}+\mathrm{CO}_{2} \rightarrow \mathrm{O}_{2}^{+}+\mathrm{CO}\right)$. Second, as illustrated by Figure $6 \mathrm{~b}$, $\mathrm{CO}_{2}^{+}$densities show a critical increase by approximately a factor of 5 above $\sim 200 \mathrm{~km}$ altitude. Besides the direct photoionization increase for $\mathrm{CO}_{2}^{+}$, the reduced loss rate also helps, which is due to reduced charge exchange collisions with atomic oxygen (see Figure 4a): $\mathrm{CO}_{2}^{+}+\mathrm{O} \rightarrow \mathrm{O}_{2}^{+}+\mathrm{CO} ; \mathrm{CO}_{2}^{+}+\mathrm{O} \rightarrow \mathrm{O}^{+}+\mathrm{CO}_{2}$. Undoubtedly, photochemical reactions become less important in competition with the transport process at such high altitudes, which, however, is neglected by 1-D photochemical models. Our work represents the first attempt to take advantage of global MHD models to consider transport processes for a dust impact study. Third, Figure $6 \mathrm{~b}$ reveals that different ionospheric species have different percentage changes in density, suggesting that the ionospheric composition is subject to important perturbations during the global storm. Fourth, after being organized using atmospheric pressure levels in Figures $6 \mathrm{c}$ and $6 \mathrm{~d}$, the ionospheric density profiles (except for $\mathrm{e}^{-}$) do not coincide with each other between the nondusty and dusty scenarios, which is remarkably different from what we have seen in Figure 5 for the regional storm. Nevertheless, the ionospheric density profiles in terms of neutral pressure do show an overlap at low altitudes. These observations suggest that the significant upper atmospheric compositional change in the severe global dust storm (see Figure 4) indeed results in a profound impact on the ionospheric densities and compositions. By considering general neutral distributions but neglecting the detailed atmospheric compositions, neutral pressures may still 
be helpful to account for the overall location change of the main ionospheric layer but fail for individual ionospheric species even in photochemical equilibrium regions.

The examination of the MHD results along the Sun-Mars line is useful to shed light on the Mars system's response to dust activities originating near the surface. However, the plasma environment at the subsolar point is subject to the maximum solar wind surppression, which in turn leads to limited vertical transport along the radial direction. To utilize the 3-D MHD model capability of self consistently simulating the solar wind-Mars interaction, we extend our analysis to make comparisons from a global perspective. Figure 7 shows the global dust-induced ionospheric impact in event 2, as a function of SZA and altitude. These 2-D density distributions are obtained by arithmetically averaging the MHD radial profiles rotating about the Sun-Mars line at any given SZA location, ranging from subsolar at $\mathrm{SZA}=0^{\circ}$ to antisubsolar at $\mathrm{SZA}=180^{\circ}$. Before examining the dust impact, we take a look at the global ionospheric distributions themselves. A few interesting ionospheric features that are not seen in a classic Chapman photochemical picture are illustrated.

First, while the ionosphere generally follows the Chapman theory below $\sim 200 \mathrm{~km}$ altitude, important deviation takes place at high altitudes. It is more prominent above $\sim 300 \mathrm{~km}$ altitude near the terminator region for all the planetary ions, shown as a rapid density increase mainly due to the combined effects of the ambipolar electric field acceleration and the $J \times B$ drag during the magnetic field draping process. There is another density bulge close to the subsolar region near $\mathrm{SZA}=10^{\circ}$, which is attributed to plumelike escaping ions under the convection electric field acceleration [e.g., Fang et al., 2008; Dong, Y., et al., 2015; Dong et al., 2018a]. Note that the ion plume is a regional feature and is aligned approximately with the convection electric field direction, which is along $+Z$ direction upstream of Mars in our simulation cases. Therefore, the plume feature in Figure 7 is not that prominent as it is (which will be discussed in more detail later), owing to our averaging manner, in which all radial profiles having the same angle from the $X$ axis are equally weighted. Furthermore, the geometry of the ion plume makes its appearing location in SZA increase with altitude, which is readily seen when examined over a greater altitude range as in Figure 8 (see particularly the density bulges shown as blue shading). 
Second, while ionospheric densities quickly drop after crossing the terminator plane and approaching the optical shadow of ionizing solar irradiance, a significant nightside ionosphere is effectively maintained even at the antisubsolar point $\left(\mathrm{SZA}=180^{\circ}\right)$. This is driven by the transterminator ionospheric flow due to the day-night plasma pressure gradient, part of which descends to replenish the nightside ionosphere that is short of photoionization. Such a day-to-night transport effect manifests itself more visibly in the $\mathrm{O}_{2}^{+}$ "tongue", which is extended throughout the entire nightside region near $150 \mathrm{~km}$ altitude in Figure 7. Note that in the MHD model, the electron impact ionization is generally considered and parameterized using thermal electrons. That is, the kinetic ionization from precipitating energetic electrons along open magnetic field lines [e.g., Lillis and Fang, 2015, and references therein] is neglected in our MHD approximations. The relative importance of particle precipitation to the day-to-night transport in the nightside ionosphere is not well understood.

The right column of Figure 7 provides a quantitative evaluation of ionospheric density changes below $400 \mathrm{~km}$ altitude on a planetary scale in response to the 1971-1972 global dust storm. Similar to what has been observed at $\mathrm{SZA}=0^{\circ}$ in Figure $6 \mathrm{~b}$, we see the altitude profiles of all ionospheric species are subject to an upward lift over the entire dayside $\left(\mathrm{SZA}<90^{\circ}\right.$ ). As a result, the densities of $\mathrm{O}_{2}^{+} / \mathrm{CO}_{2}^{+} / \mathrm{e}^{-}$above their peak altitudes increase and those below the peaks decrease. Among them, $\mathrm{CO}_{2}^{+}$has the most pronounced increase, which is up to a factor of 5 , over a broad altitude range. Moreover, their percentage changes on the dayside show a moderate SZA dependence. The patterns are more consistent below $\sim 200 \mathrm{~km}$ altitude (where the Chapman photochemical approximation is reasonable): being maximum at $\mathrm{SZA}=0^{\circ}$ and then gradually decreasing with SZA. Ionospheric $\mathrm{O}^{+}$ions show distinctly different characteristics from the other planetary ions. The $\mathrm{O}^{+}$density on average decreases over all altitude levels (see the discussions of Figure 6), and the degree of reduction below $\sim 200 \mathrm{~km}$ increases with SZA. Above $\sim 250 \mathrm{~km}$ altitude, where transport processes start to dominate over photochemical processes, the relative dust impact exhibits complex features in the SZA dependence.

Besides the dayside impact, the plasma environment on the nightside is also disturbed by the global dust storm but more importantly in terms of a relative difference. Unlike an overall upwelling of the dayside ionosphere in response to the upper atmospheric expansion, the impact on the nightside shows a dramatically different characteristics given that photoionization by solar EUV becomes insignificant toward the optical 
shadow. In consistence with the MHD perspective that the nightside ionosphere is maintained by transterminator and descending ionospheric fluxes, the nightside dust-induced ionospheric impact shows a clear connection with what happens on the dayside but not apparently with the ambient atmospheric change. Because ionospheric densities on the nightside are considerably lower than on the dayside by orders of magnitude, the enhancement factors are greatly amplified as shown in Figure 7 when more ionospheric species transport from the dayside and inject into the nightside. Accordingly, we see the percentage change gradually increases away from the terminator region and reaches the maxima near the antisubsolar point. The relative increases in the nightside density are as high as a factor of 6 for $\mathrm{O}_{2}^{+}$and $\mathrm{e}^{-}$and up to two orders of magnitude for the minor species of $\mathrm{CO}_{2}^{+}$.

It is a natural expectation that the tight coupling between the ionosphere and magnetosphere enables the upward propagation of dust-induced, low-altitude perturbations into the magnetosphere. By self-consistently simulating plasma transport within the context of the Mars-solar wind interaction, the MHD model goes beyond the limitation of simplistic photochemical approximations and offers a comprehensive global view. Figure 8 presents similar results as Figure 7 but in a much broader altitude range of up to 2000 $\mathrm{km}$. To guide the understanding of the plasma flow distribution and its interaction with the solar wind, we derive and superpose in the figure the location of the bow shock (BS) and induced magnetospheric boundary (IMB) by applying the algorithm of Fang et al. [2015, 2017]. It is seen that the BS is approximately at $2000 \mathrm{~km}$ altitude at the subsolar point for this specific case (as indicated). This is also reflected by the proton density enhancement when the solar wind is slowed down, compressed, and heated downstream of the BS. It should be pointed out that 3-D MHD results enable the determination of global boundary shapes and locations with spatial asymmetry, as demonstrated by Fang et al. [2015, 2017]. However, in order to be consistent with the reduced 2-D plot in Figure 8, we make axial symmetric conic section fits and project the average boundary locations for the reference purpose. Our results suggest that the boundaries in general show insensitivity even toward a strong global dust storm. It is found that the dust impact at high altitudes is concentrated mostly on plasma densities but little on the dynamics (i.e., velocity) or magnetic field distributions.

As illustrated in the right column of Figure 8, the dust-induced plasma density disturbance occupies a very broad spatial domain, spanning not only the ionosphere but also 
the magnetosphere. However, perturbations in electron density (i.e., total ion density) are mostly confined inside the ionosphere, which may explain in part why the high-altitude IMB and BS are barely affected. For individual ion species, on the other hand, the dust impact is extended by means of plasma transport from the ionosphere to the magnetosphere, from the dayside to the nightside, but great changes are basically limited and bounded by the IMB. Note that the large percentage differences of planetary ion densities outside of the IMB on the dayside (particularly near subsolar) are less important, given that solar wind protons are dominant in density by orders of magnitude. As a shielding barrier that weakens the solar wind penetration [e.g., see Figure 5 of Fang et al., 2015], the IMB together with the nearby piled-up magnetic field effectively inhibit upward propagation of the dust-induced disturbance. Figure 8 clearly demonstrates the enormous complexity of the dust storm consequences in the near-Mars plasma environment and the need for a global Mars-solar interaction model (like what we are using) to make assessments from a system's perspective.

Figure 9 reorganizes the comparison of the MHD results for dust event 2 using atmospheric pressure levels (see Figure 4). These assessments of the dust effects are an extension of what we have examined along the subsolar line in Figures $6 \mathrm{c}$ and $6 \mathrm{~d}$ to cover the entire global domain. A few features stand out, consistent with what have been discussed before. First, within the low-altitude atmosphere of pressure greater than $\sim 10^{-7}$ $\mathrm{Pa}$ (approximately $200 \mathrm{~km}$ altitude), the ionospheric electron density on the dayside is subject to an insignificant change in the atmospheric pressure coordinate frame. In contrast, the dayside dust impact on the densities of individual planetary ion species remains important and is different from each other, although the strength of the relative change is greatly reduced from what has been seen in Figures 7 and 8 in the altitude coordinate frame. Second, in these photochemical equilibrium regions, the dust-induced percentage changes show little dependence with SZA at pressure levels, on the contrary to the apparently strong SZA dependence at altitude levels in Figure 7. It is illustrated that the dayside main ionospheric layer reacts to the solar absorption and photoionization location change in accordance with the upper atmospheric expansion, in which plasma transport is not efficient and photochemical reactions determine the vertical structure of ionospheric ion abundances. Owing to significant atmospheric compositional changes, the ionospheric composition is greatly altered, regardless of whether altitude or neutral pressure grids are used. Third, at higher altitudes, the upper atmosphere becomes insensitive to dust activi- 
ties (Figure 4) and plasma transport dominates over photochemical reactions. As a result, the importance of local atmospheric concentrations drops, and using neutral pressure levels is not helpful in explaining the dust impact. Fourth, the nightside ionospheric disturbance cannot be accounted for in terms of neutral pressures. This is because under the MHD approximations, the nightside ionosphere is replenished by ions that are of the dayside origin and are carried across the terminator by the day-to-night transport. That is, the nightside ionospheric disturbance is more closely connected with the counterpart on the dayside than with the ambient neutral perturbation.

To have a straightforward picture of the dust-induced global consequences, we show in Figure 10 a 3-D view of the Martian ionosphere and magnetosphere under nondusty and dusty atmospheric conditions. The $\mathrm{e}^{-}$density distribution is presented at $140 \mathrm{~km}$ altitude, which is approximately the ionospheric peak location at subsolar under the dusty condition (see Figure 6a and Figure 7). As expected from axial symmetric setup of the atmospheric and solar irradiance conditions about the Sun-Mars line, we see an overall symmetry inside the ionospheric photochemical equilibrium region. The relatively weak but noticeable asymmetric features are attributed to the combined influence from the intrinsic asymmetry due to multifluid MHD approximations together with the highly nonuniformly distributed crustal magnetic field. It has been found that the crustal field has a control on the dayside ionospheric density distribution [e.g., Andrews et al., 2015]. It is readily seen from the comparison of the nondusty and dusty results that the $\mathrm{e}^{-}$density is greatly enhanced, particularly near subsolar. Also superposed in Figure 10 are planar cuts of the $\mathrm{CO}_{2}^{+}$density distributions inside the ionosphere and magnetosphere and beyond (specifically on the dayside). The most prominent feature is the dayside plume-like ion population on the meridional $(X-Z)$ plane, which is distinct from the tailward-escaping population. Accelerated by the convection electric field, which is aligned with the $+Z$ direction upstream of Mars, ion plume represents an important nonthermal ion escape channel for atmospheric erosion [e.g., Fang et al., 2008; Dong, Y., et al., 2015] and is captured by our MHD multifluid approximations [Dong et al., 2018a]. Note that the IMF direction setup in this work makes the MSO and MSE coordinate systems coincide. The comparison in Figure 10 reveals that the $\mathrm{CO}_{2}^{+}$density remarkably increases due to the global dust storm, inside not only the ionosphere but also the magnetosphere, including both the plume and tail population regions. 


\subsection{Dust Impact on Atmospheric Loss}

The density disturbances at high altitudes as illustrated in Figures 8 and 10 suggest an important implication of dust storms for total planetary ion loss. We make an assessment of how the amounts of escaping ions react to the global dust storm. The comparison is conducted in Figure 11, sufficiently far away from Mars $\left(r=6 R_{M}\right)$ to ensure that outward moving particles are lost to space. The two ion escape channels are prominent in Figure 11: the plume escape over the polar region (mainly on the dayside and swept through the nightside) and the downstream escape of plasma sheet particles (concentrated near the nightside meridional plane). The detailed comparison in the right column of Figure 11 reveals that all of these major planetary heavy ions suffer important escaping flux perturbations at least locally, resulting in net relative changes in the integrated loss amounts: $-5.6 \%\left(\mathrm{O}_{2}^{+}\right),-32.6 \%\left(\mathrm{O}^{+}\right)$, and $161.6 \%\left(\mathrm{CO}_{2}^{+}\right)$. The important dust impact on the total loss of $\mathrm{O}^{+}$and $\mathrm{CO}_{2}^{+}$are consistent with what we have seen in the magnetosphere in Figure 8, in support of the picture that dust-induced perturbations are propagated upward by plasma transport processes.

While MHD-estimated oxygen escape in the form of ions is minor in comparison with neutral escape through dissociative recombination of molecular ions $\mathrm{O}_{2}^{+}$and $\mathrm{CO}_{2}^{+}$ [e.g. Lee et al., 2015a], the net increase of $\mathrm{MHD} \mathrm{CO}_{2}^{+}$escape by a factor of $\sim 2.6$ as shown here has profound implications. According to the work of Groller et al. [2014], the neutral carbon loss rate is about $7.9 \times 10^{23} \mathrm{~s}^{-1}$ for low solar activity, the condition comparable to the solar irradiance conditions of our current MHD simulations (see section 3.1). Another independent work by Lee et al. [2015b] gives a total carbon loss estimate of $9.7 \times 10^{23} \mathrm{~s}^{-1}$ under perihelion and low solar conditions. The estimate of the carbon loss by Cui et al. [2019] is $10^{24} \mathrm{~s}^{-1}$ on average, under low to moderate solar conditions. Taking the neutral loss into account and artificially assuming a stable level of about $9 \times 10^{23} \mathrm{~s}^{-1}$, our results imply that the strong global dust storm may increase the total carbon loss (in the forms of neutrals and ions) from $1.0 \times 10^{24} \mathrm{~s}^{-1}$ to $1.2 \times 10^{24} \mathrm{~s}^{-1}$, which amounts to a relative increase of $20 \%$. However, this represents a lower bound estimate and the potential increase for total carbon loss may have been greater, given that neutral loss itself probably increases as well. In association with upper atmospheric expansion, more $\mathrm{CO}$ molecules are expected, and thus more hot carbon atoms are produced from photodissociation [Fox \& Bakalian, 2001]. When assessing the implication of global dust storms for atmospheric loss, we need to consider another equally important factor, i.e., time scale. Note that global 
dust storms typically last for months (see Figures 1 and 2), and the work of Liemohn et al. [2012] suggests that the dust impact on the upper atmosphere may last even longer by up to 7 months. All together, Mars global dust storms are more than just a powerful meteorological phenomenon. Its potential importance in atmospheric evolution (particularly for carbon loss) should be further studied.

\section{Discussion and Conclusion}

In summary, we apply the state-of-the-art multifluid MHD model to investigate how the dust-induced upper atmospheric perturbations transfer to the surrounding plasma environment over a global scale. A broad spatial domain of the model (above $100 \mathrm{~km}$ altitude) is examined, including not only the main ionosphere (which suffers direct influence due to photochemical reactions and tight neutral-ion coupling) but also the induced magnetosphere (which is more subject to indirect influence through plasma transport processes). By choosing the 2017 late-winter regional dust storm and the stronger 1971-1972 global dust storm for our case studies, our quantitative evaluation enables an assessment of the range of potential dust consequences. The discussions above focus more on the analysis of the MHD results for the 1971-1972 global dust storm. The impact of the 2017 latewinter regional dust storm has been similarly analyzed and included in the supporting information of this paper. It is found that important dust consequences also happen in the ionosphere and magnetosphere during the regional storm, but to a less degree as expected. The impact of the regional dust storm on planetary ion loss is basically negligible.

It should be stressed that dust storms are typically characterized with different timing (season), duration, spatial coverage, and magnitude (see Figure 1), and at the same time the corresponding upstream solar wind, IMF, and solar irradiance conditions are largely stochastic. In this respect, dust storms are different and somehow unique from each other, not only in dust activities themselves but also in the background atmospheric and solar conditions. To add to the complexity of modeling the dust impact on the nearMars space environment, localized crustal magnetic anomalies are an important factor. The crustal field distribution in the MSO coordinate system is determined by the seasondependent Mars' orientation to the Sun (see Figure 2) and its continuous rotation, significantly contributing to the complexity and variability of the solar wind-Mars interaction [Fang et al., 2010, 2015, 2017; Ma et al., 2014]. 
In this study, which represents the first attempt to theoretically predict the dust impact on a global scale including both the ionosphere and magnetosphere, we make a few simplified approximations. For example, we rely on previously published results to describe the atmospheric profiles under nondusty and dusty conditions, which serve as input to the MHD model. Except for background atmospheric conditions, all the other driving factors are held identical in our controlled MHD runs. The model runs until a quasi steady state is reached; by this means, we neglect fluctuations over short periods of time. Nevertheless, by using conditions as physically realistic as possible, it suffices for us to perform a first-order assessment of whether and how dust storms could extend their effects thousands of kilometers from the bottom side of the ionosphere into space. It is left to future study to include self-consistently configured 3-D atmospheres (including thermal and hot components) and allow for time variation of the system and its drivers.

It is found that the ionosphere can be significantly disturbed during dust storms. On the dayside, the net effect on ionospheric vertical profiles of electron densities or total ion densities basically is upwelling of the main ionospheric layer (below $\sim 250 \mathrm{~km}$ altitude). The peak altitudes are upward lifted by $\sim 5 \mathrm{~km}$ and $\sim 15 \mathrm{~km}$ for the regional and global dust storm, respectively. This occurs nearly uniformly on the dayside, in accordance with the assumed expansion of the entire upper atmosphere. Similar vertical shifts happen to individual planetary $\mathrm{O}_{2}^{+} / \mathrm{CO}_{2}^{+} / \mathrm{O}^{+}$ion distributions, although relatively light $\mathrm{O}^{+}$ions (which peak at high altitudes) are subject to a greater elevation. During ionospheric upwelling, there is little change in the peak densities of electrons and ions. As an exception, the whole $\mathrm{O}^{+}$density profile may be significantly reduced in the global dust storm, due to more severe ion loss through charge exchange collisions with enhanced ambient $\mathrm{CO}_{2}$ neutrals. Consistent with the upward lift, charged particle densities increase (decrease) at altitudes above (below) their peak locations. The ionospheric composition is significantly altered in the global storm, as a result of dust-induced perturbations in the neutral composition. In contrast, the ionospheric composition basically is stable during the regional storm. In these photochemical equilibrium regions (below $\sim 250 \mathrm{~km}$ ), using neutral pressure levels in place of altitude levels is helpful to explain the ionospheric upwelling in response to the regional storm, but fails in the case of the global storm except for electron density profiles. This supports that photochemical reactions still play a dominant role in determining the main ionospheric layer, but sufficiently large compositional changes in neutrals would in turn result in noticeable ion mixing ratio changes. 
Unlike the direct and straightforward reactions of the dayside ionosphere to the upper atmospheric perturbations, the nightside ionosphere responds to the dust storm in an indirect sense. From the MHD perspective, the source of the nightside ionosphere comes from the plasma of the dayside origin, which is carried by the day-to-night transport across the terminator and then descends to low altitudes. As a result, the nightside ionospheric change shows a close connection with what happens on the dayside but not apparently with the ambient atmospheric change. Because of considerably lower plasma abundances on the nightside, the percentage change due to dust storms is greatly amplified in comparison with the dayside part. Consistent with the day-to-night transport, the maximum relative change appears deep in the optical shadow, even over orders of magnitude. However, it has been suggested by previous studies [e.g., Nemec et al., 2010; Duru et al., 2011; Cui et al., 2015; Girazian et al., 2017; Adams et al., 2018] that particle precipitation constitutes an important, direct ionization source to the ionosphere in addition to the dayto-night transport, particularly far into the nightside. In this scenario, dust-induced atmospheric expansion on the nightside would more effectively prevent particle penetration and therefore enhance energy deposition and particle impact ionization rates at higher altitudes and reduce them at lower altitudes. The resulting upward shift of the nightside ionosphere due to particle precipitation would be mixed with the indirect changes that we have seen in this study due to the day-to-night transport. Untangling the relative importance of particle precipitation and transterminator transport, with the crustal magnetic field taken into account, is an important topic for future work.

At altitudes higher than $\sim 250 \mathrm{~km}$, the transport process becomes important and takes over the control of plasma distributions from local photochemical reactions. As a result, we see that dust-induced perturbations propagate upward from the ionosphere to the magnetosphere, and extend from the dayside to the nightside. While the electron density or the total ion density seems to have their disturbances limited to altitudes below $\sim 500$ $\mathrm{km}$, the densities of planetary ions $\left(\mathrm{O}_{2}^{+}, \mathrm{CO}_{2}^{+}, \mathrm{O}^{+}\right)$react to the dust storms throughout the entire magnetosphere generally bounded by the IMB. This suggests that the IMB and the nearby magnetic field pileup not only weaken the solar wind penetration but also constitute a barrier to effectively inhibit the upward propagation of low-altitude perturbations. Despite probable strong local changes to densities at high altitudes, the total ion escape rates are hardly impacted during the regional storm. On the contrary, the total loss of $\mathrm{O}_{2}^{+}$ and $\mathrm{CO}_{2}^{+}$in the global storm may change by $-32.6 \%$ and $161.6 \%$, respectively. Taking 
neutral carbon loss into account, our results imply that total carbon loss, in the forms of neutrals (mainly through photodissociation of $\mathrm{CO}$ ) and ions (through $\mathrm{CO}_{2}^{+}$in this study), may be subject to a net increase of $\sim 20 \%$ or higher during strong dust storms. Considering that global dust storms are an event over a time scale of months and their disturbances on the upper atmosphere may last even longer [Liemohn et al., 2012], this work suggests that the potential importance of intense dust storms in Mars atmospheric evolution needs further study.

\section{Acknowledgments}

The work was supported by the NASA MAVEN project through the Mars Exploration Program. Resources supporting the MHD simulation were provided by the NASA HighEnd Computing Program through the NASA Advanced Supercomputing Division at Ames Research Center. The MHD simulation code is publicly available at http://csem.engin.umich.edu/tools/swmf.

The development of multi-annual dust scenarios from multi-satellite observations is funded by the French Centre National d'Études Spatiales (CNES), and the latest updated scenarios are available through the Mars Climate Database (MCD) project at http://wwwmars.lmd.jussieu.fr.

\section{References}

Adams, D., Xu, S., Mitchell, D. L., Lillis, R. J., Fillingim, M., Andersson, L., et al. (2018). Using magnetic topology to probe the sources of Mars' nightside ionosphere. Geophysical Research Letters, 45, 12,190-12,197. https://doi.org/10.1029/2018GL080629.

Andrews, D., L. Andersson, G. Delory, R. Ergun, A. Eriksson, C. Fowler, T. McEnulty, M. Morooka, T. Weber, and B. Jakosky (2015), Ionospheric plasma density variations observed at Mars by MAVEN/LPW, Geophys. Res. Lett., 42, 8862-8869, doi:10.1002/2015GL065241.

Bougher, S. W., Murphy, J., and Haberle, R. M. (1997), Dust storm impacts on the Mars upper atmosphere, Advances in Space Research, 19(8), 1255-1260. https://doi.org/10.1016/S0273âĂ ̌̌1177(97)00278â̆̌̆̃o.

Bougher, S. W., G. Keating, R. Zurek, J. Murphy, R. Harberle, J. Hollingsworth, , and R. Clancy (1996), Mars Global Surveyor aerobraking: atmospheric trends and model interpretation, Advances in Space Research, 23, 1887-1897. 
Bougher, S. W., D. A. Brain, J. L. Fox, F. Gonzalez-Galindo, C. Simon-Wedlund, and P. G. Withers (2017), Chapter 14: Upper Atmosphere and Ionosphere, in The Atmosphere and Climate of Mars, ed. B. Haberle, M. Smith, T. Clancy, F. Forget, R. Zurek, Cambridge University Press, doi:10.1017/9781107016187.

Cantor, B. A., P. B. James, M. Caplinger, and M. J. Wolff (2001), Martian dust storms: 1999 Mars Orbiter Camera observations, J. of Geophys. Res., 106(E10), 23,653-23,687, doi:10.1029/2000JE001310.

Christensen, P. R., et al. (2001), Mars Global Surveyor Thermal Emission Spectrometer experiment: Investigation description and surface science results, J. Geophys. Res., 106, $23,823-23,871$.

Christensen, P. R., et al. (2004), The Thermal Emission Imaging System (THEMIS) for the Mars 2001 Odyssey Mission, Space Sci. Rev., 110, 85-130.

Clancy, R., M. Wolff, B. Whitney, B. Cantor, M. Smith, and T. McConnochie (2010), Extension of atmospheric dust loading to high altitudes during the 2001 Marsdust storm: MGS TES limb observations, Icarus, 207, 98-109.

Cui, J., Galand, M., Yelle, R. V., Wei, Y., and Zhang, S. J. (2015), DayâĂ̌̆toâĂ ̌̌night transport in the Martian ionosphere: Implications from total electron content measurements. J. Geophys. Res. Space Physics, 120, 2333âĂŞ 2346. doi: 10.1002/2014JA020788.

Cui, J., Wu, X.âĂ̌̆S., Gu, H., Jiang, F.âĂ̌̌Y., \& Wei, Y. (2019). Photochemical escape of atomic $\mathrm{C}$ and $\mathrm{N}$ on Mars: Clues from a multiâĂ Řinstrument MAVEN dataset. Astronomy \& Astrophysics, 621, A23. https://doi.org/10.1051/0004-6361/201833749.

Dong, C., Bougher, S. W., Ma, Y., Lee, Y., Toth, G., Nagy, A. F., et al. (2018a), Solar wind interaction with the Martian upper atmosphere: Roles of the cold thermosphere and hot oxygen corona, Journal Geophysical Research: Space Physics, 123, 6639-6654. https://doi.org/10.1029/2018JA025543

Dong, C., et al. (2018b), Modeling Martian Atmospheric Losses over Time: Implications for Exoplanetary Climate Evolution and Habitability, ApJ Letters, 859, L14.

Dong, Y., X. Fang, D. A. Brain, J. P. McFadden, J. S. Halekas, J. E. Connerney, S. M. Curry, Y. Harada, J. G. Luhmann, and B. M. Jakosky (2015), Strong plume fluxes at Mars observed by MAVEN: An important planetary ion escape channel, Geophys. Res. Lett., 42, doi:10.1002/ 2015GL065346. 
Duru, F., Gurnett, D. A., Morgan, D. D., Winningham, J. D., Frahm, R. A., and Nagy, A. F. (2011), Nightside ionosphere of Mars studied with local electron densities: A general overview and electron density depressions, J. Geophys. Res., 116, A10316, doi:10.1029/2011JA016835.

Eparvier, F., P. Chamberlin, T. Woods, and E. Thiemann (2015), The solar extreme ultraviolet monitor for MAVEN, Space Sci. Rev., doi:10.1007/s11214-015-0195-2.

Fang, X., M. W. Liemohn, A. F. Nagy, Y. Ma, D. L. De Zeeuw, J. U. Kozyra, and T. H. Zurbuchen (2008), Pickup oxygen ion velocity space and spatial distribution around Mars, J. Geophys. Res., 113, A02210, doi:10.1029/2007JA012736

Fang, X., M. W. Liemohn, A. F. Nagy, J. G. Luhmann, and Y. Ma (2010a), On the effect of the martian crustal magnetic field on atmospheric erosion, Icarus, 206, 130-138, doi:10.1016/j.icarus.2009.01.012.

Fang, X., Y. Ma, D. Brain, Y. Dong, and R. Lillis (2015), Control of Mars global atmospheric loss by the continuous rotation of the crustal magnetic field: A time-dependent MHD study, J. Geophys. Res. Space Physics, 120, 10,926-10,944, doi:10.1002/2015JA021605.

Fang, X., et al. (2017), The Mars crustal magnetic field control of plasma boundary locations and atmospheric loss: MHD prediction and comparison with MAVEN, J. Geophys. Res. Space Physics, 122, doi:10.1002/2016JA023509.

Forget, F., F. Montmessin, J.-L. Bertaux, F. Gonzalez-Galindo, S. Lebonnois, E. Quemerais, A. Reberac, E. Dimarells, and M. A. Lopez-Valverde (2009), Density and temperatures of the upper Martian atmosphere measured by stellar occultations with Mars Express SPICAM, J. Geophys. Res.,114, E01004, doi:10.1029/2008JE003086.

Fox, J. L. (2004), Response of the Martian thermosphere/ionosphere to enhanced fluxes of solar soft X rays, J. Geophys. Res., 109, A11310, doi:10.1029/2004JA010380.

Fox, J. L., \& Bakalian, F. M. (2001). Photochemical escape of atomic carbon from Mars. Journal of Geophysical Research, 106(A12), 28,785âĂŞ28,795. https://doi.org/10.1029/2001JA000108

Girazian, Z., Mahaffy, P. R., Lillis, R. J., Benna, M., Elrod, M., and Jakosky, B. M. (2017), Nightside ionosphere of Mars: Composition, vertical structure, and variability, J. Geophys. Res. Space Physics, 122, 4712âĂŞ 4725, doi:10.1002/2016JA023508.

Groller, H., H. Lichtenegger, H. Lammer, and V. Shematovich (2014), Hot oxygen and carbon escape from the martian atmosphere, Planetary and Space Science, 98, 93-105, 
doi:10.1016/j.pss.2014.01.007.

Haberle, R. M., C. B.Leovy, and J. B. Pollack (1982), Some effects of global dust storms on the atmospheric circulation of Mars, Icarus, 50, 322-367.

Haider, S. A., V. Sheel, M. D. Smith, W. C. Maguire, and G. J. MolinaâĂ̌̆Cuberos (2010), Effect of dust storms on the D region of the Martian ionosphere: Atmospheric electricity, J. Geophys. Res., 115, A12336, doi:10.1029/2010JA016125.

Hantsch, M. H., and S. J. Bauer (1990), Solar control of the Mars ionosphere, Planet. Space Sci., 38, 539-542.

Jakosky, B., et al. (2015), The Mars Atmosphere and Volatile EvolutioN (MAVEN) mission, Space Sci. Rev., 195, 3, doi:10.1007/s11214-015-0139-x.

Kahre, M., J. Murphy, C. Newman, R. Wilson, B. Cantor, M. Lemmon, M. Wolff (2017), The Mars Dust Cycle. In R. Haberle, R. Clancy, F. Forget, M. Smith, \& R. Zurek (Eds.), The Atmosphere and Climate of Mars (Cambridge Planetary Science, pp. 295337). Cambridge: Cambridge University Press. doi:10.1017/9781139060172.010.

Keating, G. M., et al. (1998), The structure of the upper atmosphere of Mars: in situ accelerometer measurements from Mars Global Surveyor, Science, 279, 1672âĂŞ1676.

Kliore, A. J., G. Fjeldbo, B. L. Seidel, M. J. Sykes, and P. M. Woiceshyn (1973), S band radio occultation measurements of the atmosphere and topography of Mars with Mariner 9: Extended mission coverage of polar and intermediate latitudes, J. Geophys. Res., 78(20), 4331-4351, doi:10.1029/JB078i020p04331.

Lee, Y., Combi, M. R., Tenishev, V., Bougher, S. W., Deighan, J., Schneider, N. M., et al. (2015a), A comparison of 3-D model predictions of MarsâĂŹ oxygen corona with early MAVEN IUVS observations, Geophysical Research Letters, 42, 9015âĂŞ9022. https://doi.org/10.1002/2015GL065291.

Lee, Y., Combi, M. R., Tenishev, V., and Bougher, S. W. (2015b), Hot carbon corona in Mars' upper thermosphere and exosphere: 2 . Solar cycle and seasonal variability, $J$. Geophys. Res. Planets, 119, 2487âĂŞ 2509, doi: 10.1002/2014JE004669.

Lee, Y., P. Mahaffy, M. Benna, S. Bougher, Y. Ma, X. Fang, C. Dong, V. Tenishev, D. Pawlowski, and M. Combi (2018), Variability of the Martian ionospheric peak and hot O corona - observations and simulation, AGU Fall Meeting Abstracts, abstract P43K3913.

Lee, Y., X. Fang, M. Gacesa, Y. Ma, V. Tenishev, P. Mahaffy, C Dong, M. Combi, S. Bougher, and B. Jakosky (2019), Effects of global and regional dust storms on the Mar- 
tian hot O corona and photochemical loss, J. Geophys. Res. Space Physics, this special issue.

Liemohn, M. W., A. Dupre, S. W. Bougher, M. Trantham, D. L. Mitchell, and M. D. Smith (2012), TimeâĂ Ǩhistory influence of global dust storms on the upper atmosphere at Mars, Geophys. Res. Lett., 39, L11201, doi:10.1029/2012GL051994.

Lillis, R. J., and X. Fang (2015), Electron impact ionization in the Martian atmosphere: Interplay between scattering and crustal magnetic field effects, J. Geophys. Res. Planets, 120, doi:10.1002/2015JE004841.

Liu, G., England, S. L., Lillis, R. J., Withers, P., Mahaffy, P. R., Rowland, D. E., et al. (2018), Thermospheric expansion associated with dust increase in the lower atmosphere on Mars observed by MAVEN/NGIMS, Geophysical Research Letters, 45, 2901âĂŞ2910, https://doi.org/10.1002/2018GL077525.

Liu, Y., A. Nagy, T. Gombosi, D. DeZeeuw, and K. Powell (1999), 3D multifluid MHD studies of the solar interaction with Mars, Geophysical Research Letters, 26, 2689.

Ma, Y., A. F. Nagy, I.V. Sokolov, and K. C. Hansen (2004), Three-dimensional, multispecies, high spatial resolution MHD studies of the solar wind interaction with Mars, $J$. Geophys. Res., 109, A07211, doi:10.1029/2003JA010367.

Ma, Y., X. Fang, C. T. Russell, A. F. Nagy, G. Toth, J. G. Luhmann, D. A. Brain, and C. Dong (2014b), Effects of crustal field rotation on the solar wind plasma interaction with Mars, Geophys. Res. Lett., 41, doi:10.1002/2014GL060785.

Mahaffy, P. R., Richard Hodges, R., Benna, M., King, T., Arvey, R., Barciniak, M., et al. (2014), The Neutral Gas and Ion Mass Spectrometer on the Mars Atmosphere and Volatile EvolutioN mission, Space Science Reviews, 185(1âĂ̌̌4), 27âĂŞ61, https://doi.org/10.1007/s11214âĂ̌̌11014âĂ ̌̌10091âĂ ̌̌11211.

McCleese, D. J., J. T. Schofield, F. W. Taylor, S. B. Calcutt, M. C. Foote, D. M. Kass, C. B. Leovy, D. A. Paige, P. L. Read, and R. W. Zurek (2007), Mars Climate Sounder: An investigation of thermal and water vapor structure, dust and condensate distributions in the atmosphere, and energy balance of the polar regions, J. Geophys. Res., 112, E05S06, doi:10.1029/2006JE002790.

Medvedev, A. S., E. Yigit, T. Kuroda, and P. Hartogh (2013), General circulation modeling of the martian upper atmosphere during global dust storms, J. Geophys. Res. Planets, 118, 1âĂŞ13, doi:10.1002/jgre.20163,2013. 
Montabone, L., F. Forget, E. Millour, R. Wilson, S. Lewis, B. Cantor, D. Kass, A. KleinbÃúehl, M. Lemmon, M. Smith, and M. Wolff (2015), EightâǍŘyear climatology of dust optical depth on Mars, Icarus, 251, 65âĂŞ95, doi:10.1016/j.icarus.2014.12.034.

Montabone, L., F. Forget (2018), Forecasting Dust Storms on Mars: A Short Review. Chapter 8 of the book Dust in the Atmosphere of Mars and Its Impact on Human Exploration, Edited by J.S. Levine, D. Winterhalter, and R.L. Kerschmann, Cambridge Scholars Publishing, UK.

Montabone, L., A. Spiga, D. Kass, A. Kleinboehl, F. Forget, and E. Millour (2019), Martian Year 34 Dust Climatology: Reconstructed Maps and Model Simulations J. Geophys. Res. Planets, this special issue.

Najib, D., A. F. Nagy, G. Toth, and Y. J. Ma (2011), Three-dimensional, multi-fluid, high spatial resolution MHD model studies of the solar wind interaction with Mars, J. Geophys. Res., 116, A05204, doi:10.1029/2010JA016272.

Nemec, F., Morgan, D. D., Gurnett, D. A., and Duru, F. (2010), Nightside ionosphere of Mars: Radar soundings by the Mars Express spacecraft, J. Geophys. Res., 115, E12009, doi:10.1029/2010JE003663.

Nemec, F., D. D. Morgan, C. DiÃlval, and D. A. Gurnett (2015), Intensity of nightside MARSIS AIS surface reflections and implications for lowâĂ Ǩaltitude ionospheric densities, J. Geophys. Res. Space Physics, 120, 3226-3239, doi: 10.1002/2014JA020888.

Schunk, R. W., and A. F. Nagy (2009), Ionospheres: Physics, Plasma Physics, and Chemistry (2nd ed.), New York: Cambridge University Press, Cambridge, U.K.

Smith, M. D., B. J. Conrath, J. C. Pearl, and P. R. Christensen (2002), Thermal emission spectrometer observations of martian planet-encircling dust storm 2001a, Icarus, 157(1), 259-263, doi:http://dx.DOI.org/10.1006/icar.2001.6797.

Toigo, A., M. Richardson, H. Wang, S. Guzewich, and C. Newman (2018), The cascade from local to global dust storms on Mars: Temporal and spatial thresholds on thermal and dynamical feedback, Icarus, 302, 514-536.

Vignes, D., et al. (2000), The solar wind interaction with Mars: Locations and shapes of the bow shock and the magnetic pile-up boundary from the observations of the MAG/ER Experiment onboard Mars Global Surveyor, Geophys. Res. Lett., 27(1), 49-52, doi:10.1029/1999GL010703.

Wang, J. S., and E. Nielsen (2003), Behavior of the Martian dayside electron density peak during global dust storms. Planetary and Space Science, 51(4âĂŞ5), 329-338. 
https://doi.org/10.1016/S0032-0633(03)00015-1.

Withers, P., and R. Pratt (2013), An observational study of the response of the upper atmosphere of Mars to lower atmospheric dust storms, Icarus, 225(1), 378âĂŞ389, https://doi.org/10.1016/j.icarus.2013.02.032

Xu, S., M. W. Liemohn, D. L. Mitchell, andM. D. Smith (2014), Mars photoelectronenergy and pitch angle dependence onintense lower atmospheric dust storms, J. Geophys. Res. Planets, 119, 1689-1706,doi:10.1002/2013JE004594.

Zhang, M. H. G., J. G. Luhmann, and A. J. Kliore (1990), An observational study of the nightside ionospheres of Mars and Venus with radio occultation methods, J. Geophys. Res., 95(A10), 17095-17102, doi:10.1029/JA095iA10p17095.

Zurek, R. W. (1982), Martian great dust storms: An update, Icarus, 50, 288-310.

Zurek, R. W., J. Barnes, R. Haberle, J. Pollack, J. Tillman, and C. Leovy (1992), Dynamics of the atmosphere of Mars, in Mars, edited by H. Kieffer et al., pp. 835-933, Univ. of Ariz., Tucson.

Zurek, R. W. and L. J. Martin (1993), Interannual variability of planet-encircling dust storms on Mars, J. Geophys. Res. 98, 3247-3259. 


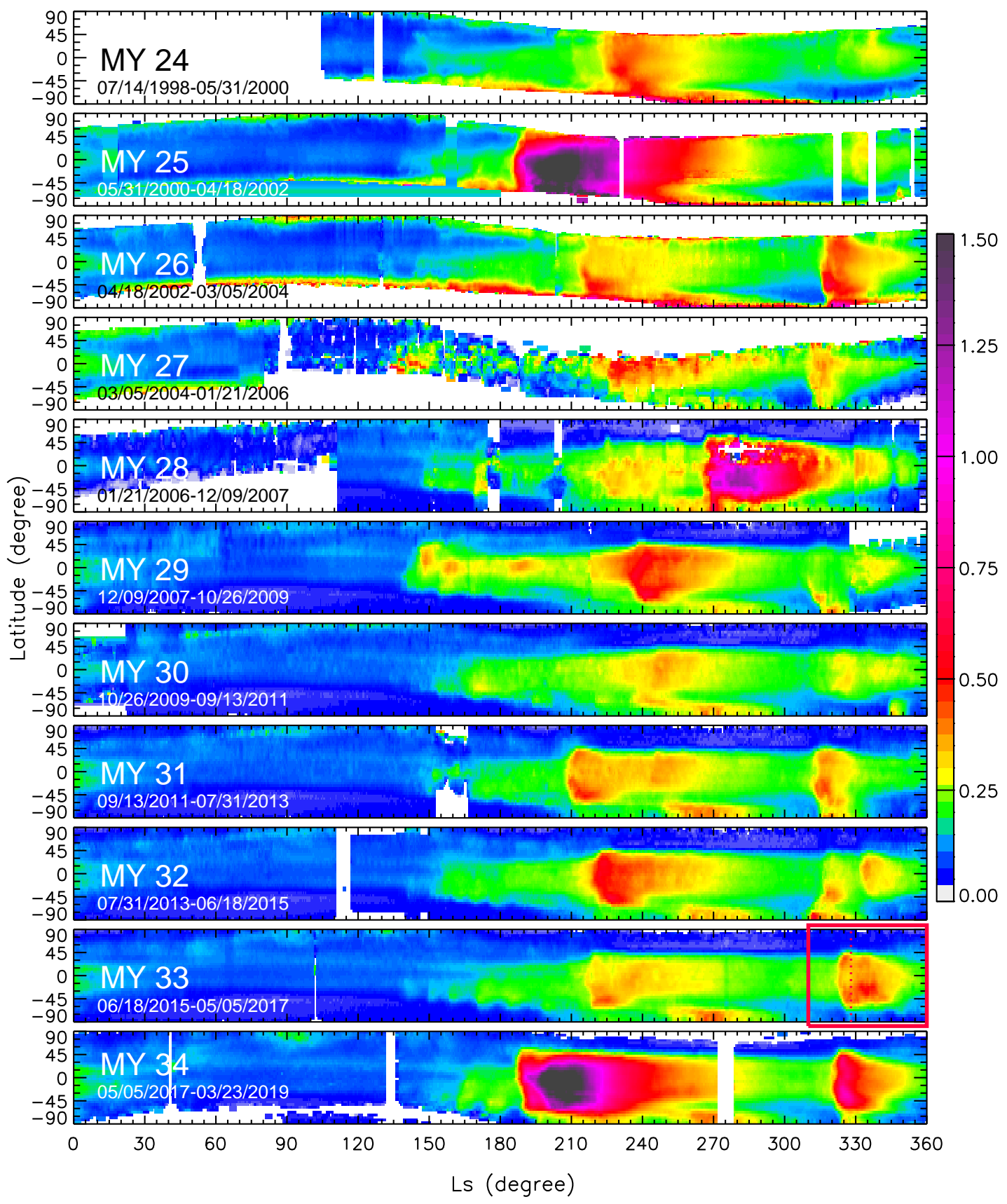

Figure 1. Mars zonally-averaged column dust optical depth at the infrared wavelength of about $9.3 \mu \mathrm{m}$ during 11 Martian years. The dust opacity, scaled to the atmospheric pressure level of $610 \mathrm{~Pa}$, is shown as a function of solar longitude $\left(\mathrm{L}_{s}\right)$ and geographic latitude. The start and end dates on Earth are given for each Martian year, and white areas indicate missing data. The red box in the second to last panel denotes the currently investigated 2017 regional dust storm period, and the vertical dashed line indicates the time when a dusty condition is taken for this study. Note that while MY 24-33 zonal means are created using the Mars Climate Database gridded dust climatology versions 2.0 and 2.1, MY 34 zonal mean is created using an updated version (as described in Montabone et al. [2019]). Therefore, caution is required when directly comparing MY 34 and the other years (particularly MYs 28-33).

This article is protected by copyrizht. All rights reserved. 


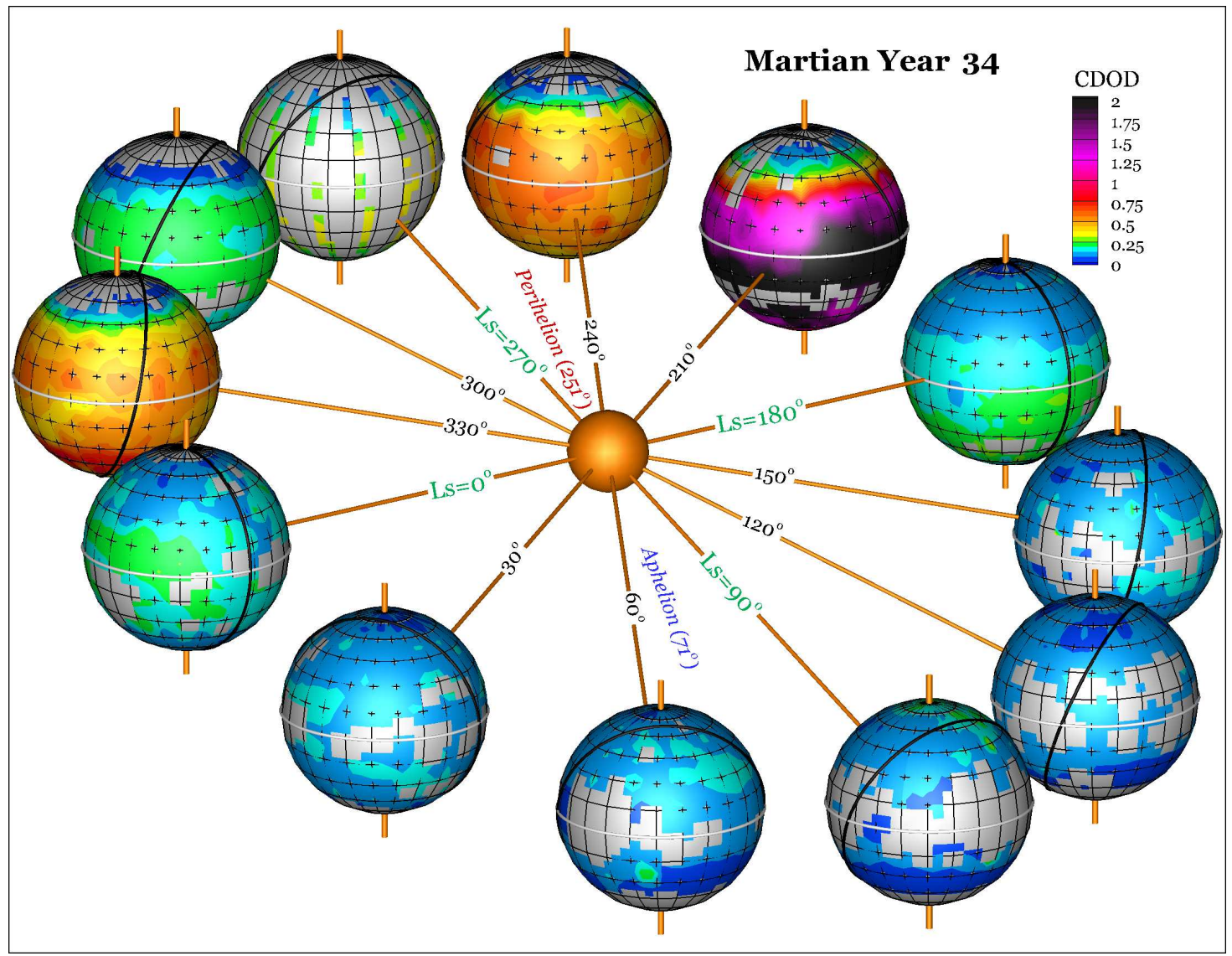

Figure 2. Global distributions of column dust optical depth at the wavelength of $9.3 \mu$ m during Martian Year 34. The dust opacity has been scaled to the atmospheric pressure of $610 \mathrm{~Pa}$. Gray areas indicate missing data. The planetary rotational axis is shown in yellow, and the equatorial and terminator circles are superposed as thick white and black curves, respectively. While the sizes of the solar and Martian bodies are not scaled in comparison with the distances between them, the planetary orientations and orbital positions are based on calculations using NAIF-SPICE. Note that the color scale is different from Figure 1. 

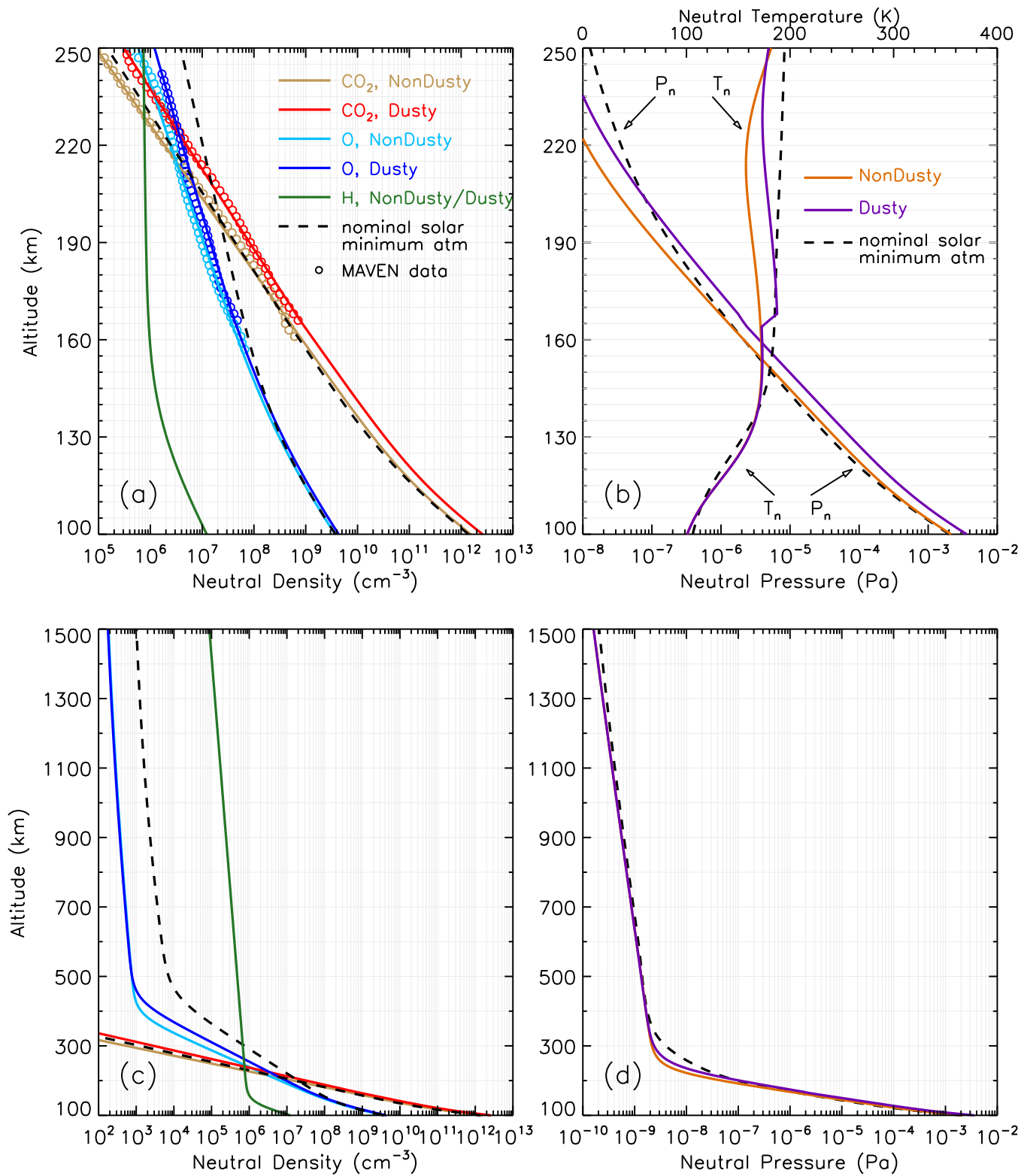

Figure 3. Altitude profiles of the upper atmospheric density (left) and temperature and pressure (right) under nondusty and dusty conditions for the simulation of the 2017 regional dust storm period (event 1 ). The circles in panel (a) represent the density of $\mathrm{CO}_{2}$ and $\mathrm{O}$ inferred from MAVEN measurements. The solid lines for these two species are our fit and extension into the simulation domain of the MHD model (see the text). The green line shows the $\mathrm{H}$ density profile, regardless of nondusty or dusty conditions, which are adopted from a typical MHD setting for a nominal solar minimum atmosphere. The dashed lines are the nominal $\mathrm{CO}_{2}$ and $\mathrm{O}$ distributions for reference. Panel (b) shows the total neutral pressure (bottom axis) and bulk neutral temperature (top axis) under nondusty and dusty conditions, with the nominal altitude profiles shown in dashed line. Panels (c) and (d) are the zoom out of panels (a) and (b), respectively, showing the hot oxygen and hydrogen coronas in an extended altitude range. 

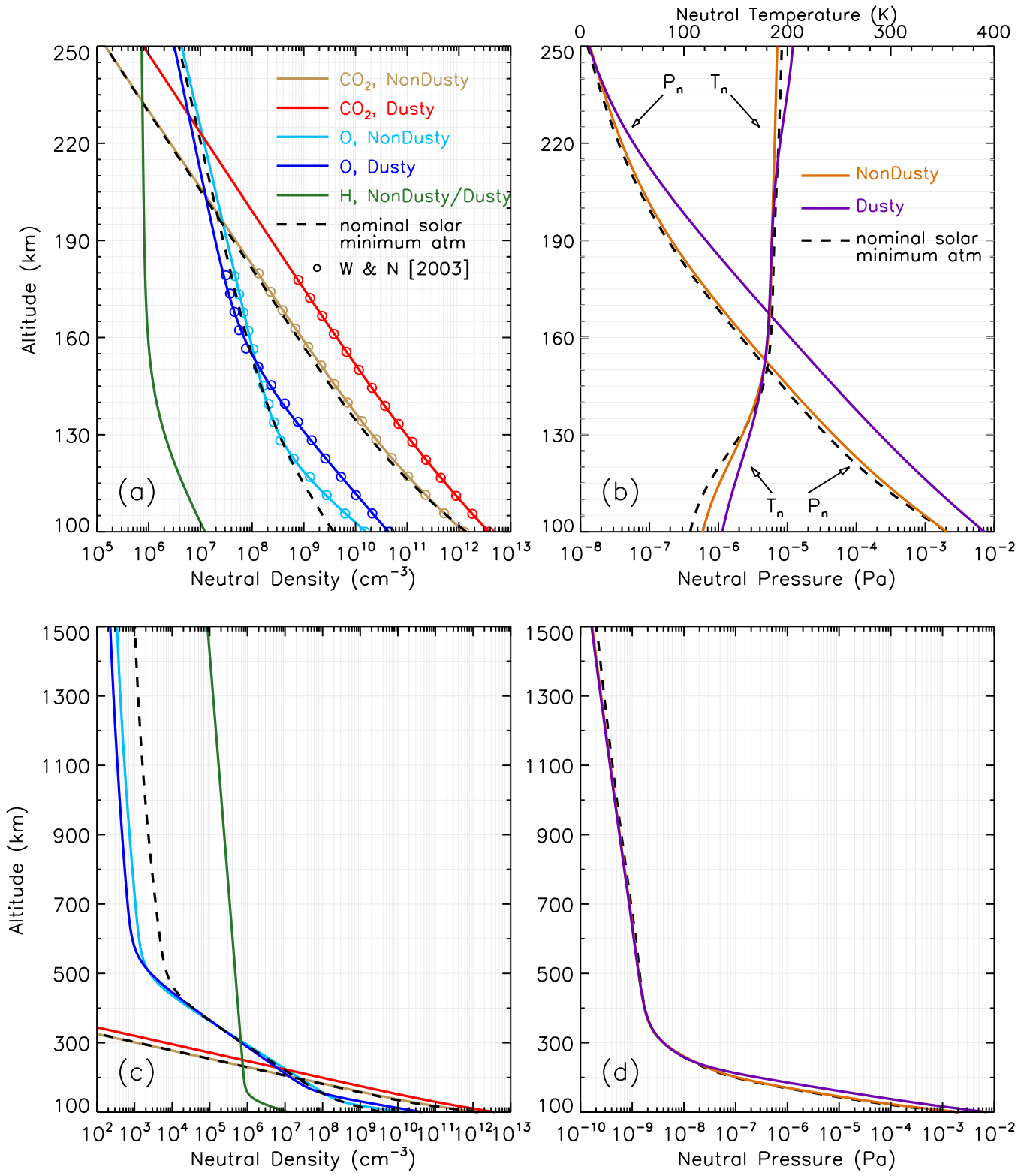

Figure 4. Similar to Figure 3 but for the 1971-1972 global dust storm period (event 2). The circles indicate the estimates obtained by Wang and Nielsen [2003]. 

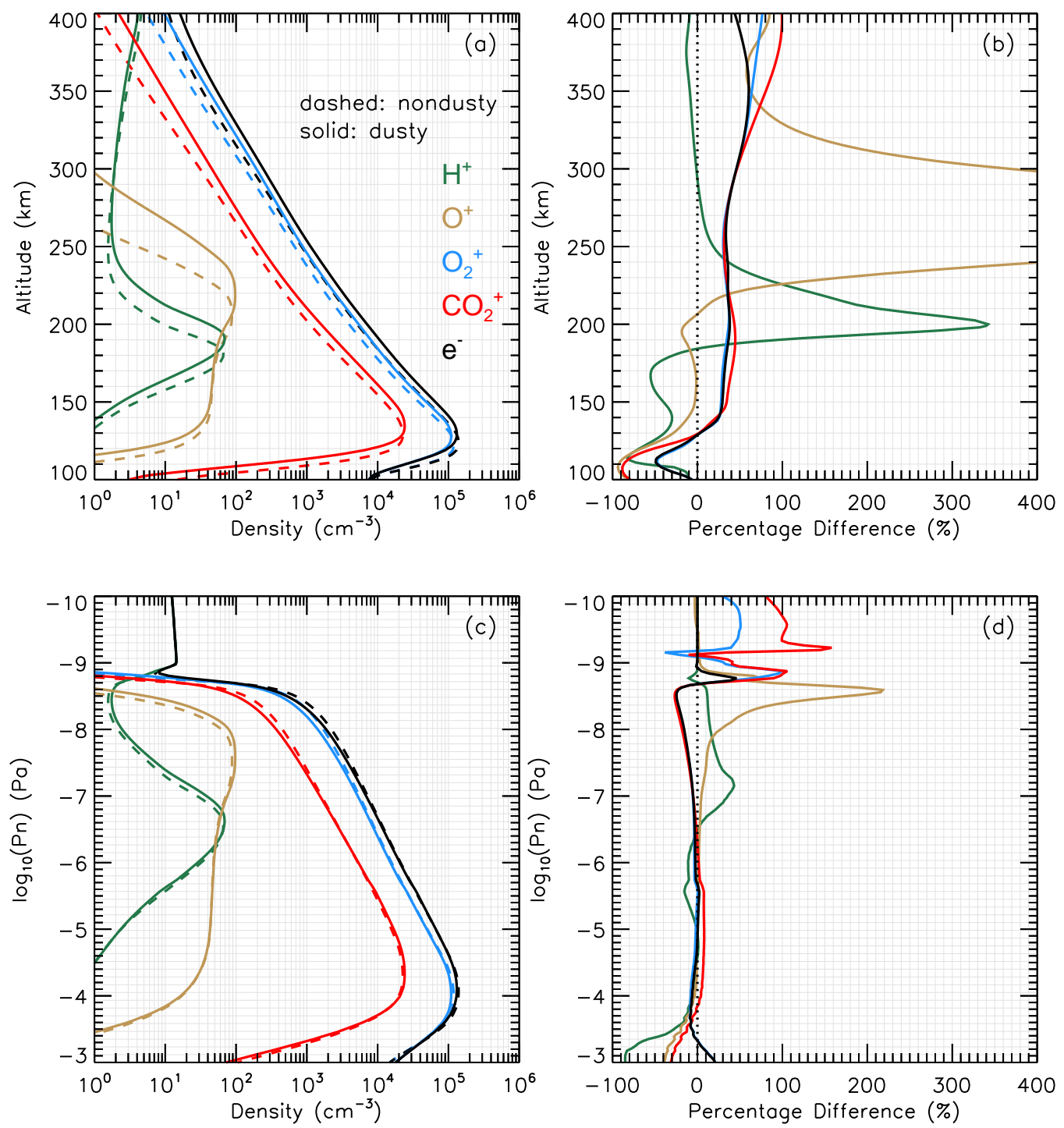

Figure 5. MHD-calculated ionospheric density disturbances along the subsolar line for dust event 1 . Panel (a) shows the altitude profiles of ion and electron densities under nondusty (in dashed) and dusty (in solid) atmospheric conditions. Panel (b) shows the percentage change of the densities due to the dust storm. The second row is similar to the first row, expect that the results are presented at atmospheric pressure levels. The pressure axis has been reversed to show increasing altitude from the bottom to the top. Also note that the pressure axis covers a much broader altitude range than in the first row. For example, the topside atmospheric pressure of $10^{-10} \mathrm{~Pa}$ is at an altitude higher than $1500 \mathrm{~km}$. 

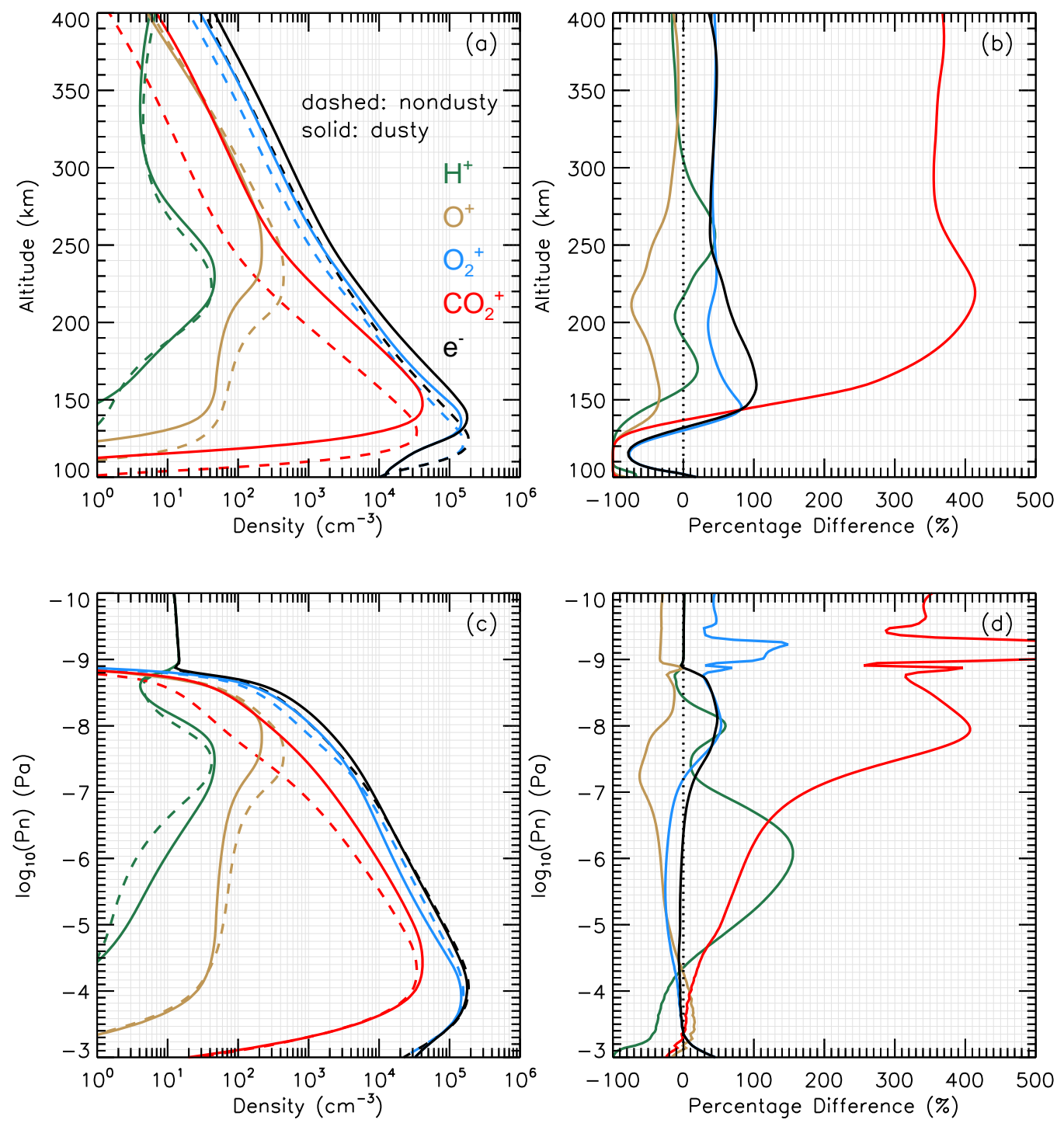

Figure 6. Subsolar ionospheric density disturbances for dust event 2, in a format similar to Figure 5. 

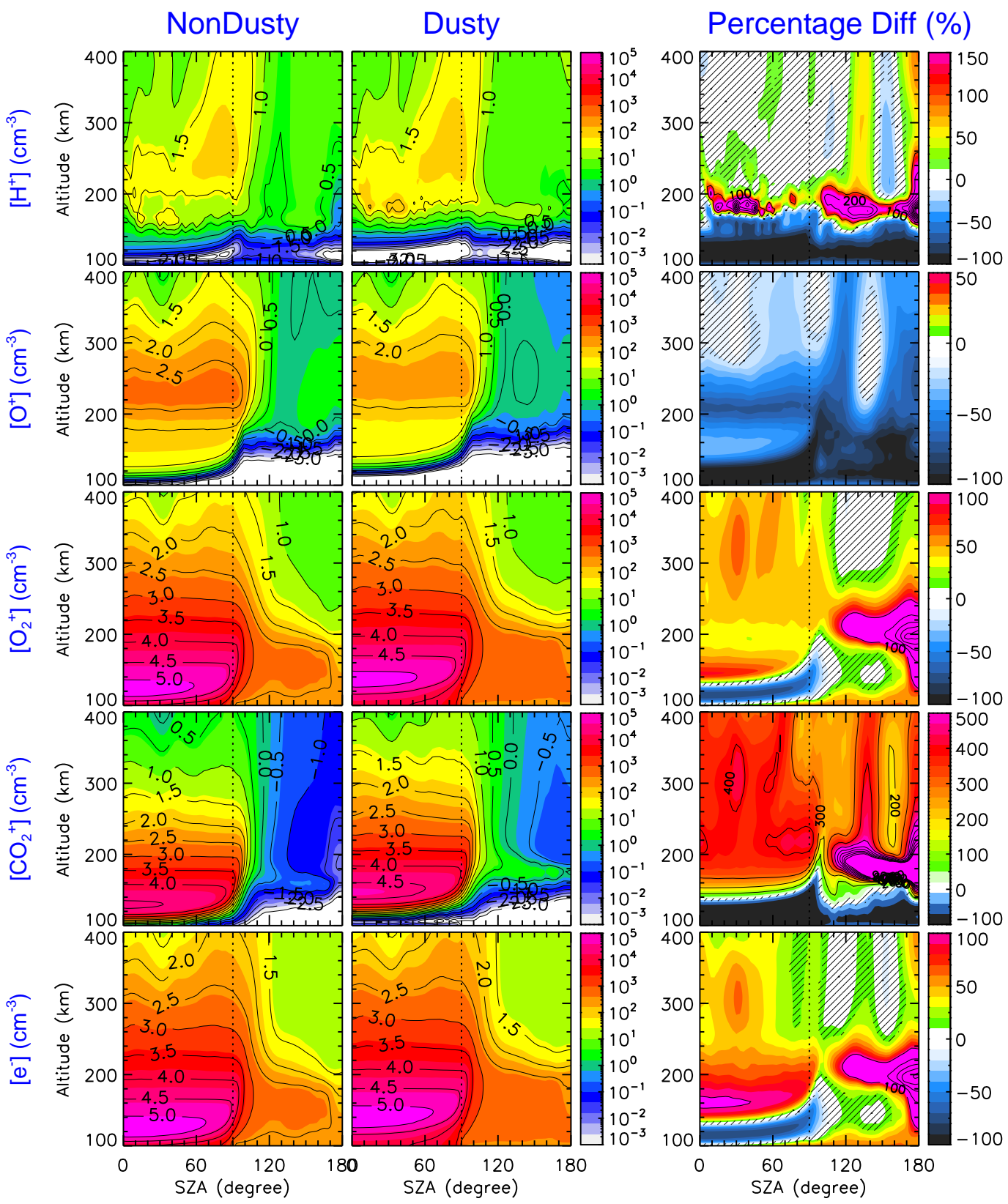

Figure 7. MHD-calculated ionospheric density disturbances for dust event 2 as a function of SZA and altitude. The left two columns present the SZA-averaged ionospheric densities under nondusty and dusty atmospheric conditions, respectively. The right column shows their percentage differences, i.e., dusty values minus nondusty divided by nondusty. From top to bottom, the panels show the results for different ionospheric species, using different color scales for the percentage changes. In the right column, the contour lines indicate a percentage change of every $100 \%$ interval, particularly useful on the nightside where the relative difference may be sufficiently high to make the color scale saturated. The hatched areas mark the places having a modest change, where the absolute percentage difference is less than $20 \%$. 

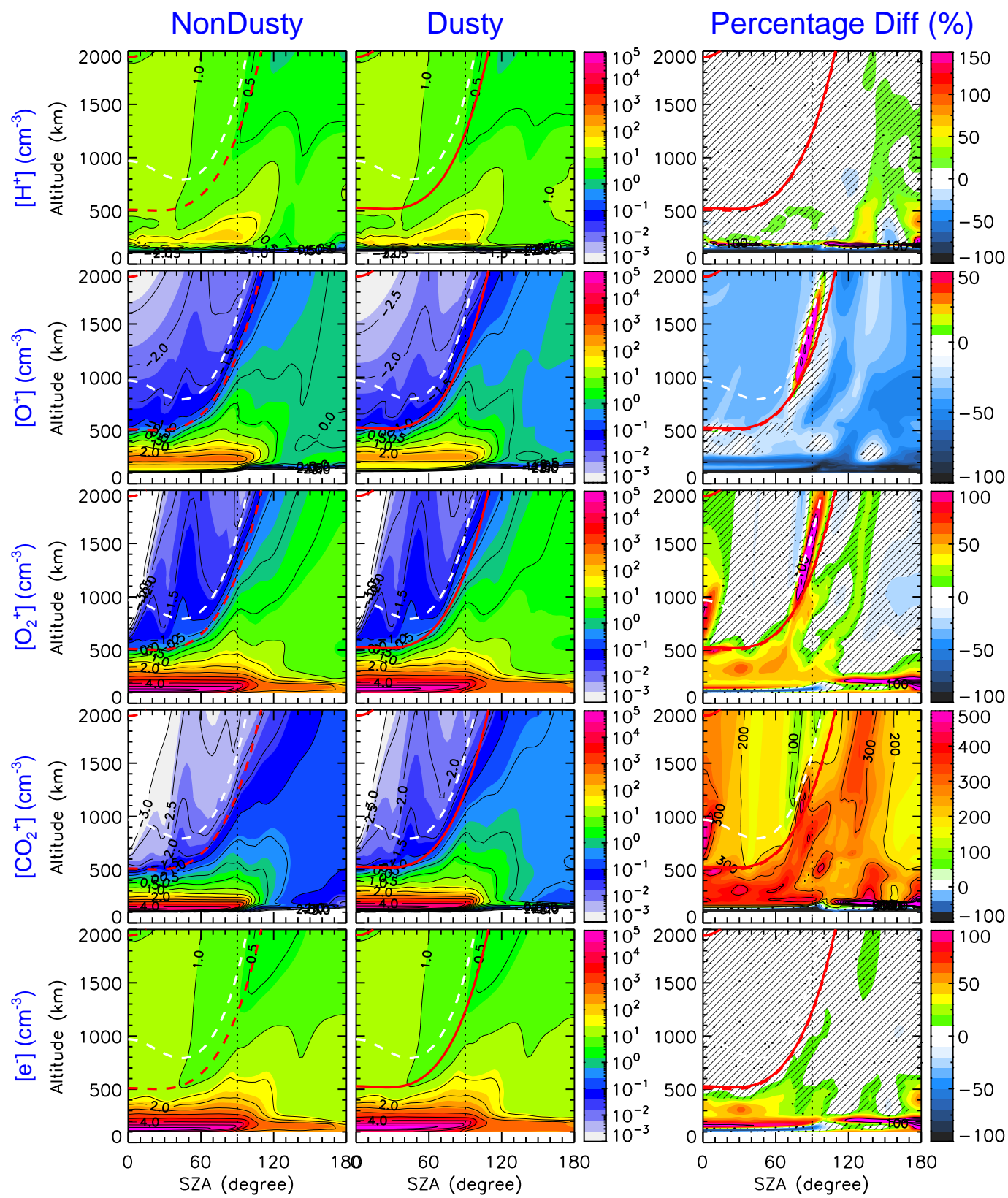

Figure 8. Similar to Figure 7 but including the results of the induced magnetosphere during dust event

2, with the altitude limit extended up to as high as $2000 \mathrm{~km}$. The average location of the induced magnetospheric boundary, which is obtained using a conic section fit, is shown as red dashed (solid) lines for nondusty (dusty) conditions. The empirical location by Vignes et al. [2000] is superposed as white dashed lines for reference. Note that our MHD-derived bow shock is also shown but partly at the upper left corners of the panels, which is located mostly higher than $2000 \mathrm{~km}$ altitude except near subsolar in this specific case. As a comparison, the lowest altitude of the empirical bow shock is $\sim 2190 \mathrm{~km}$. 

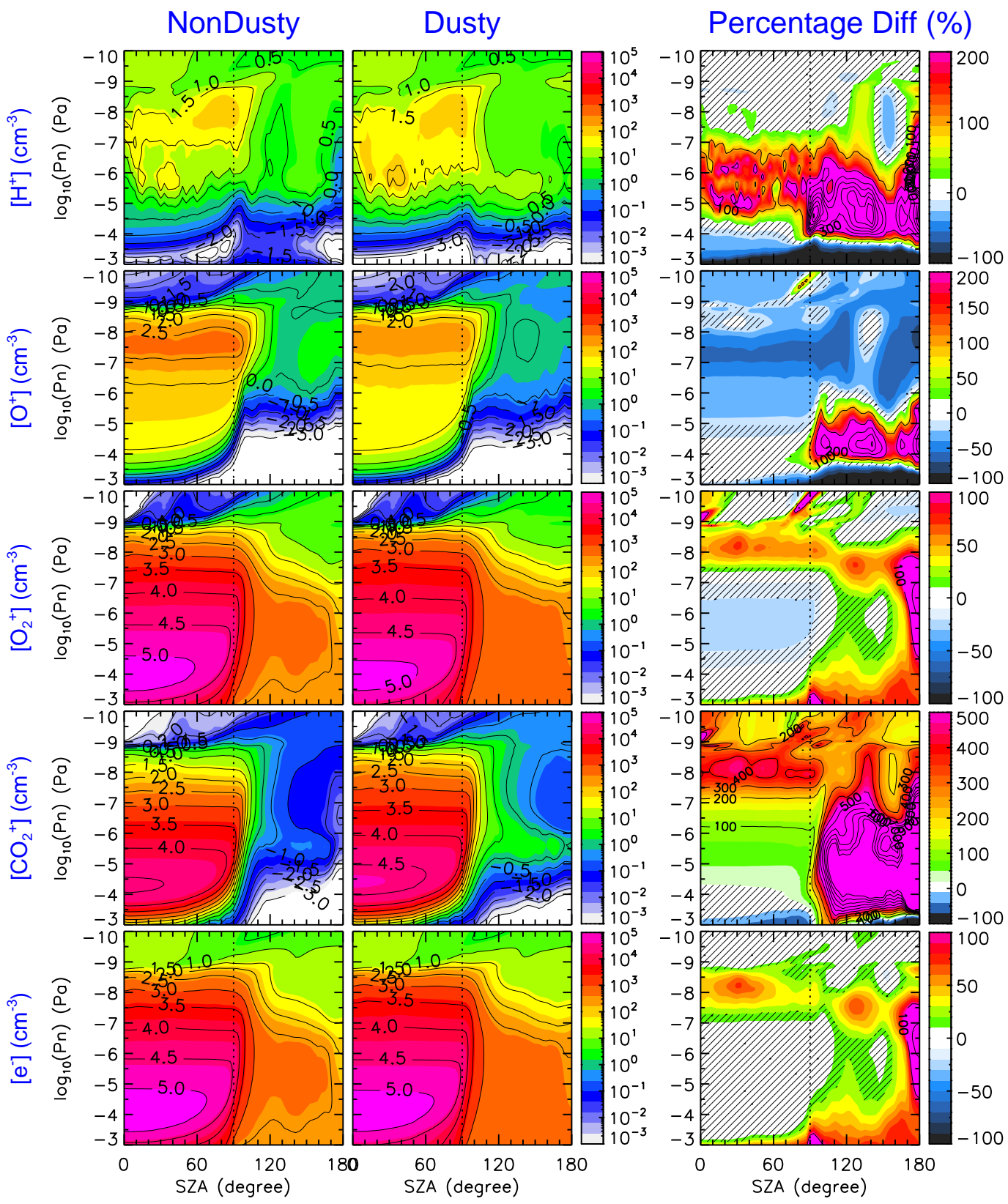

Figure 9. Similar to Figures 7 and 8 except that the results for dust event 2 are presented at atmospheric pressure levels. The pressure axis has been reversed in correspondence with altitude increase from the bottom to the top. 

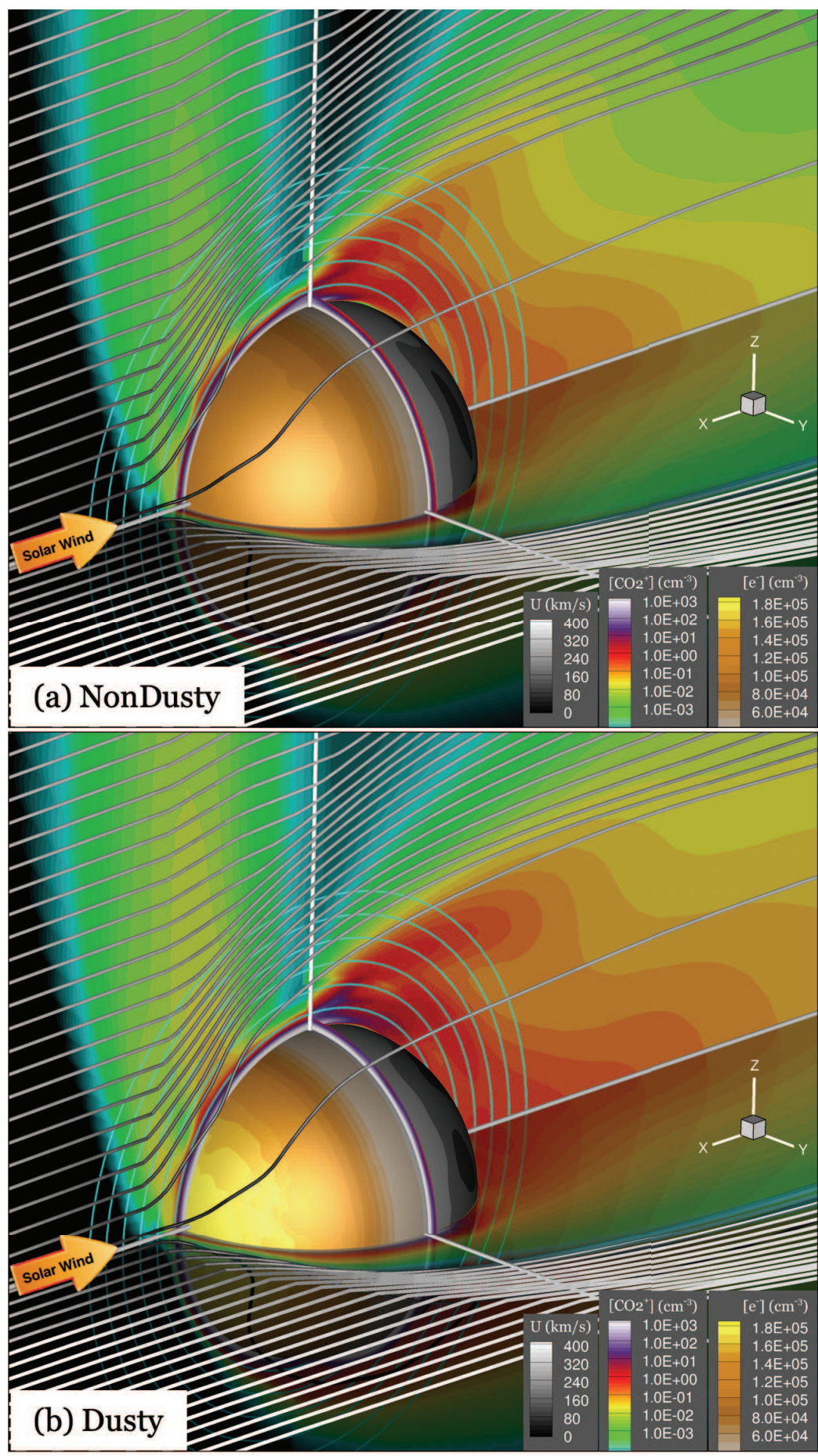

Figure 10. The 3-D view of the Martian ionospheric and magnetospheric disturbances during dust event 2 by comparing the MHD results under (left) nondusty and (right) dusty atmospheric conditions. The gray curves show streamlines of mass-averaged plasma flow (color coded by the speed), originating in the upstream on the MSO $X-Z$ (meridional) and $X-Y$ (equatorial) planes. The spherical surface shows the ionospheric $\mathrm{e}^{-}$ density at $140 \mathrm{~km}$ altitude. On the $X-Z, X-Y$, and $Y-Z$ (terminator) planes, we superpose the color contours of the $\mathrm{CO}_{2}^{+}$density. Note that the $\mathrm{CO}_{2}^{+}$distribution on the terminator plane is shown up to $400 \mathrm{~km}$ altitude in order not to block the view. The cyan concentric circles on the $X-Z$ plane indicate the altitudes from $500 \mathrm{~km}$

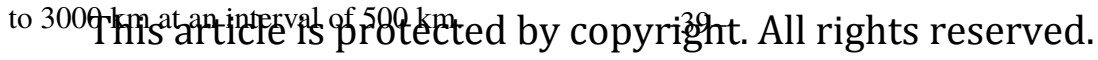



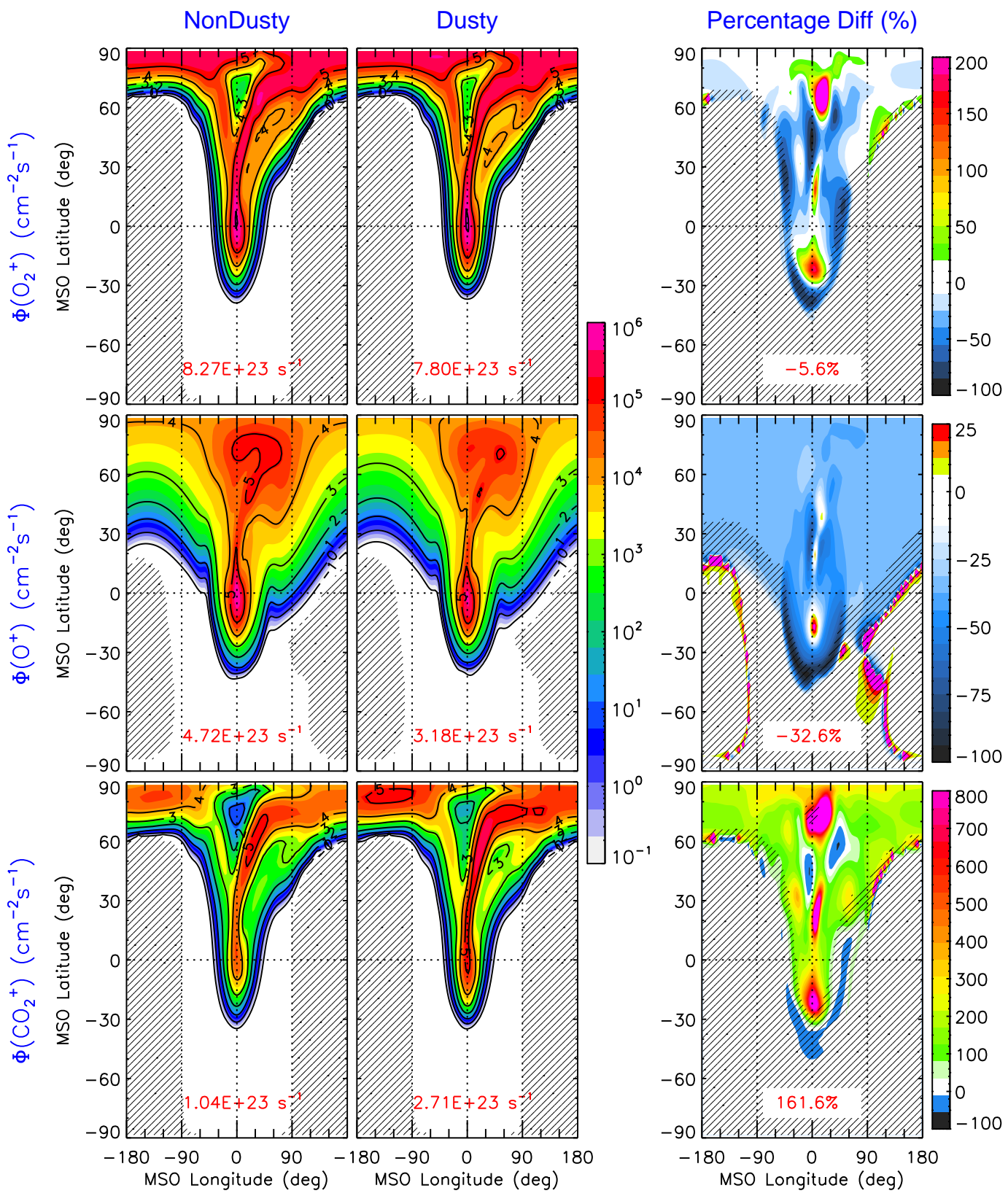

Figure 11. Comparison of the MHD-calculated planetary ion fluxes escaping through the spherical surface at a radial distance of $6 R_{M}$ from the Mars center during dust event 2. The panels from top to bottom show the results for $\mathrm{O}_{2}^{+}, \mathrm{O}^{+}$, and $\mathrm{CO}_{2}^{+}$, respectively. The left two columns present the results under nondusty and dusty atmospheric conditions, respectively, as a function of MSO longitude and latitude. The MSO latitude is measured from the MSO equatorial plane, on which $0^{\circ}$ longitude and $\pm 180^{\circ}$ longitude point toward the antisunward and sunward directions, respectively. The hatched areas mark negative fluxes, that is, for ion velocities having a radially inward component. The spherically integrated loss rates are indicated on the bottom of the panels. In the right column, we show the percentage difference between the left two columns. The hatched areas correspond to insignificant ion fluxes of less than $10 \mathrm{~cm}^{-2} \mathrm{~s}^{-1}$, where the relative comparison is less meaningful. The percentage differences of the total loss rates are indicated on the panels. 
的 MY 24

-45 - $07 / 14 / 1998-05 / 31 / 2000$

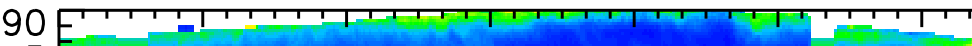

$45=$

OE MY 25

$-45=05 / 31 / 2000-04 / 18 / 2002$

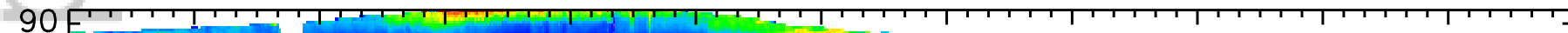
45

(2) WV 26

$-45=04 / 18 / 2002-03 / 05 / 2004$

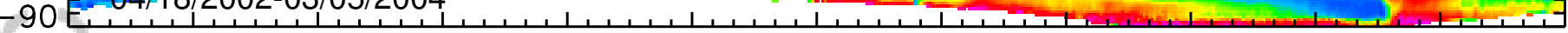

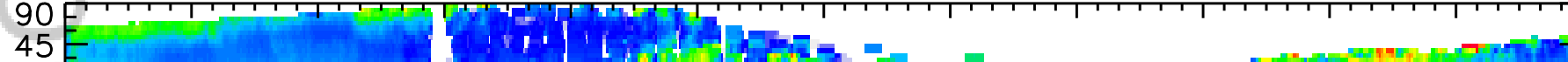

C 0 E MV 27

$-45=03 / 05 / 2004-01 / 21 / 2006$

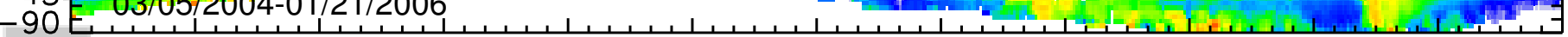

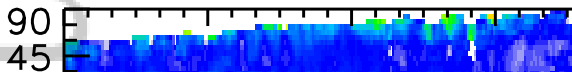

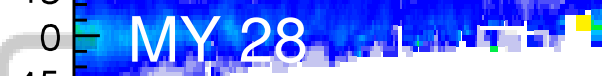

(1) $-45=01 / 2172006-12 / 09 / 2007$

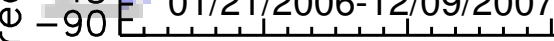

O

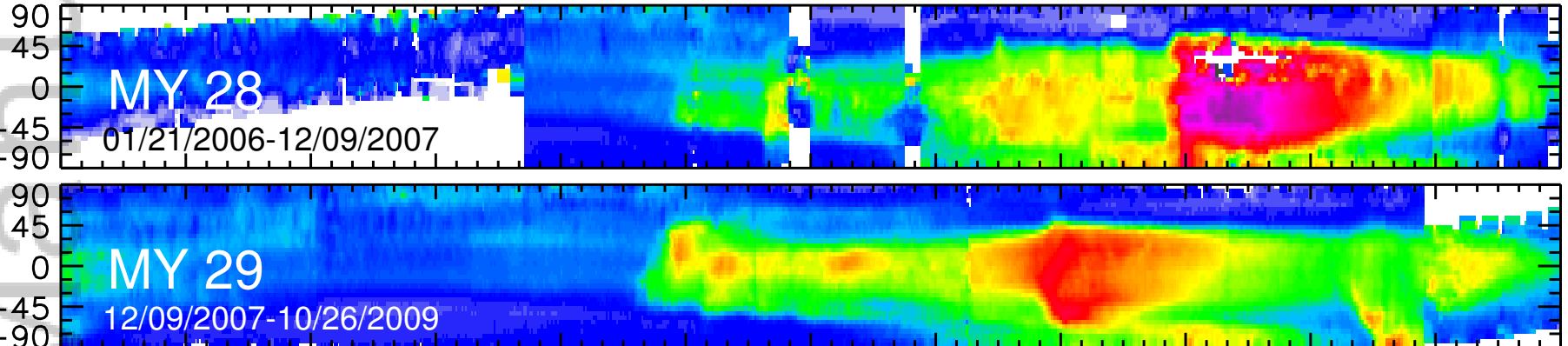

$-90$

90

45

$0=$ MY 30

$-45-10 / 26 / 2009-09 / 13 / 2011$

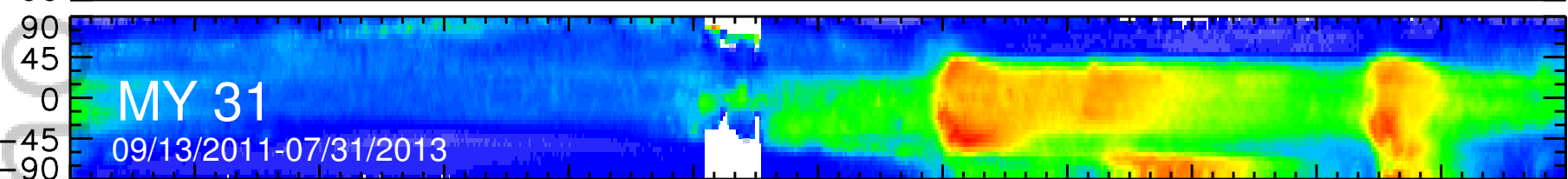

$-90=09 / 13 / 2011-07 / 31 / 2013$

90

45

OE MY 32

$-45=07 / 31 / 2013-06 / 18 / 2015$

$-90$

90

45

OE MY 33

$-45=06 / 18 / 2015-05 / 05 / 2017$

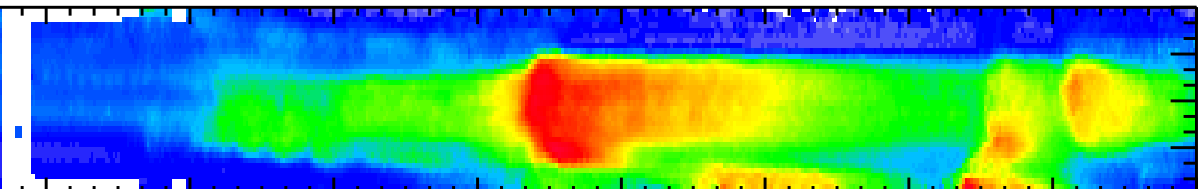

0.75

1.00

0.50

90

0 E MY 34

-45 E 05/05/2017-03/23isarticle is protected by copyright. All rights reserved.

$-90 \mathrm{E}, \mathrm{H}-1$ 
Figure 2.
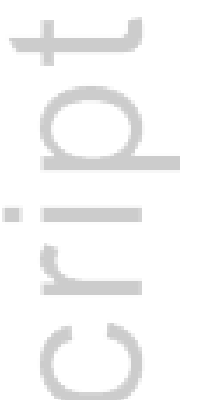

$\infty$
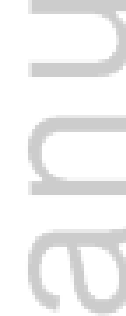

This article is protected by copyright. All rights reserved. 
Figure 5.
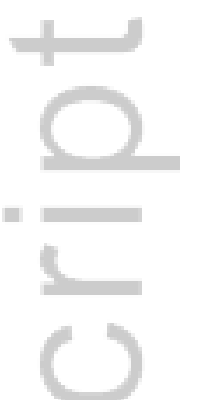

$\infty$
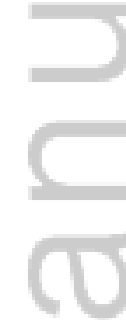

This article is protected by copyright. All rights reserved. 

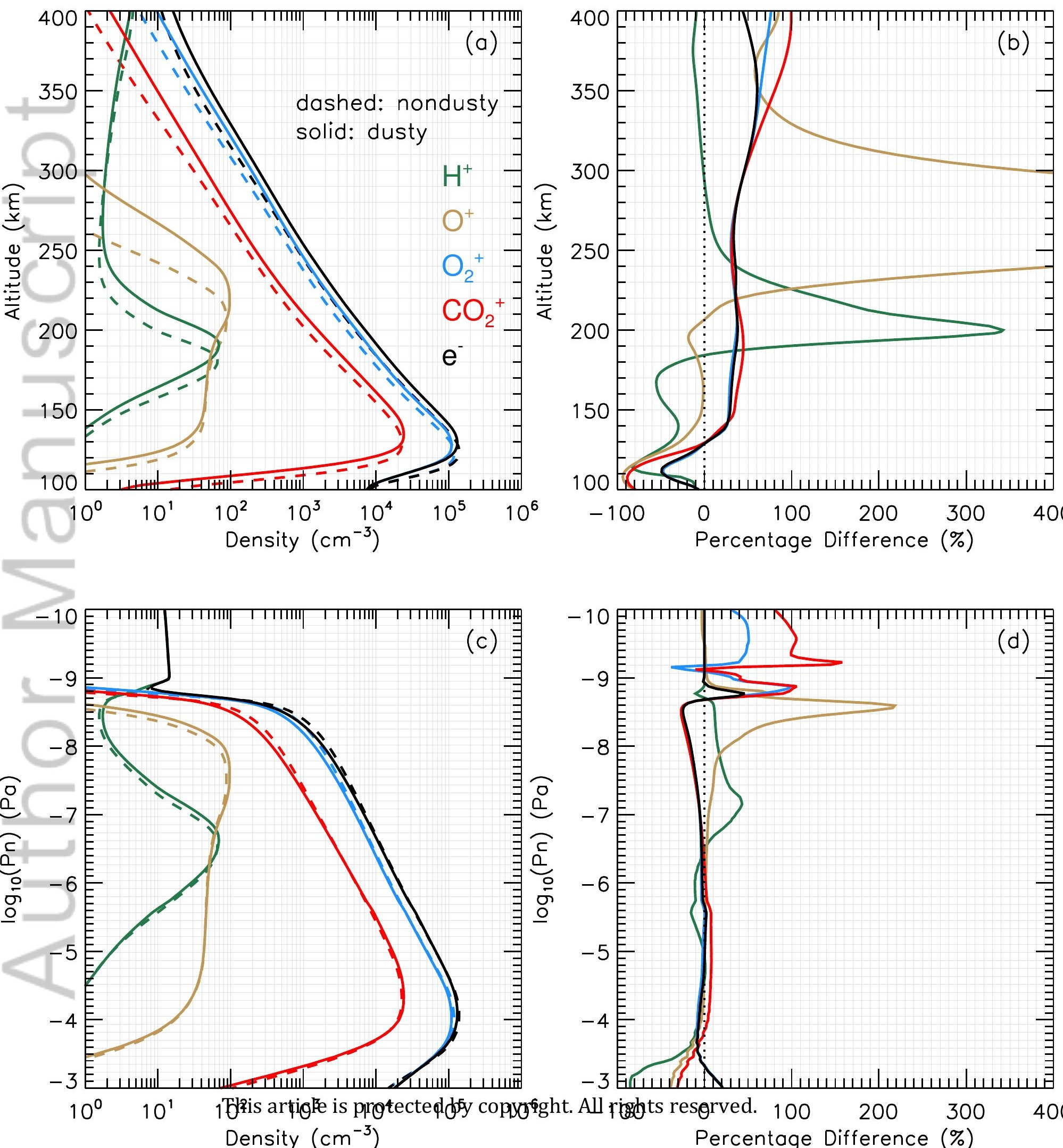


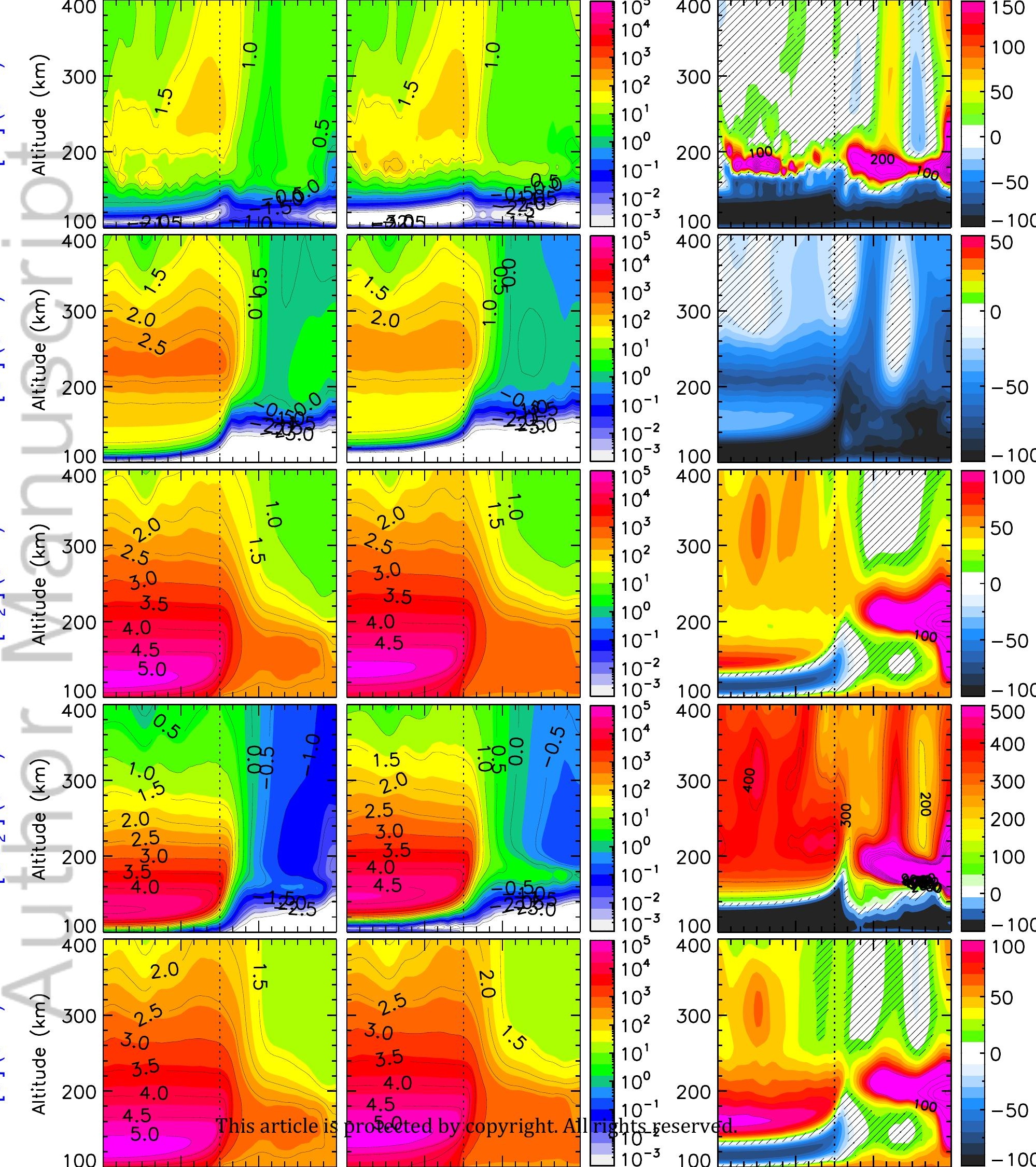


Figure 9.
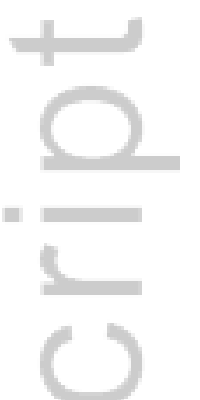

$\infty$
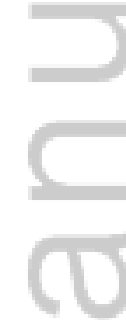

This article is protected by copyright. All rights reserved. 


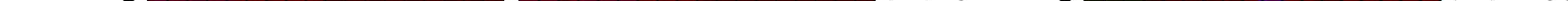



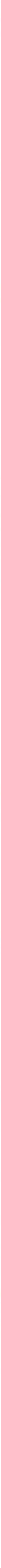


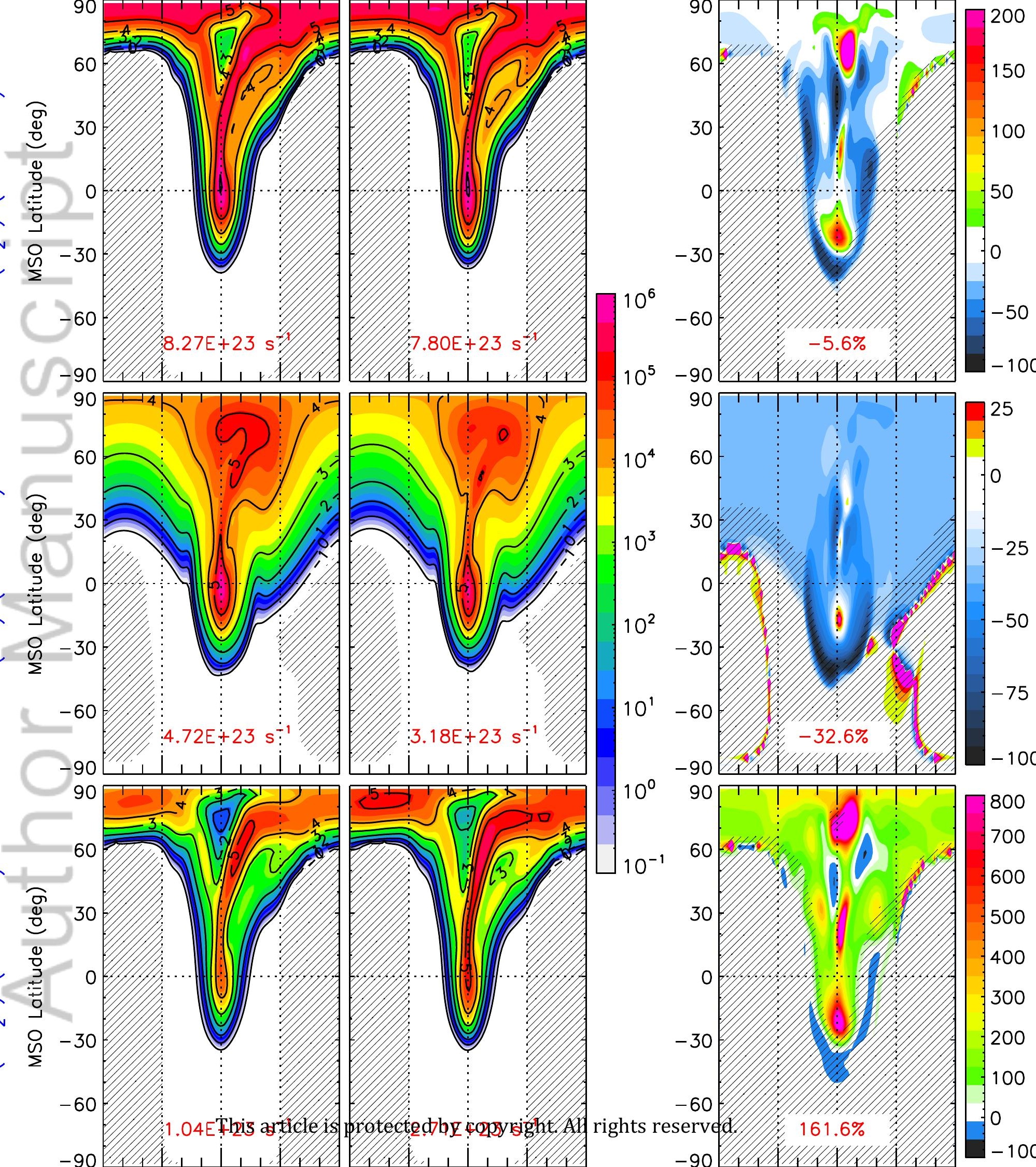




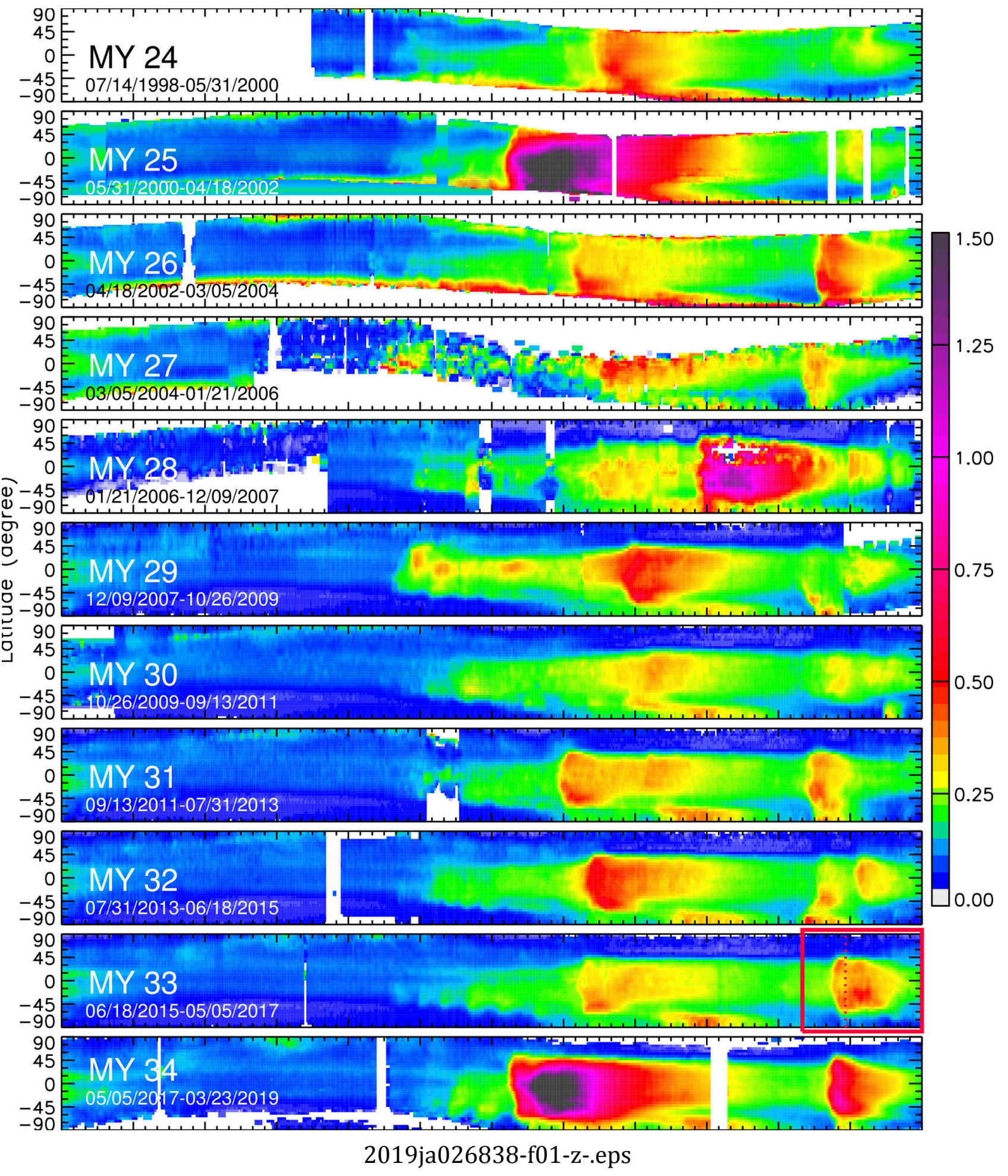

This article is protected by copyright. All rights reserved. 


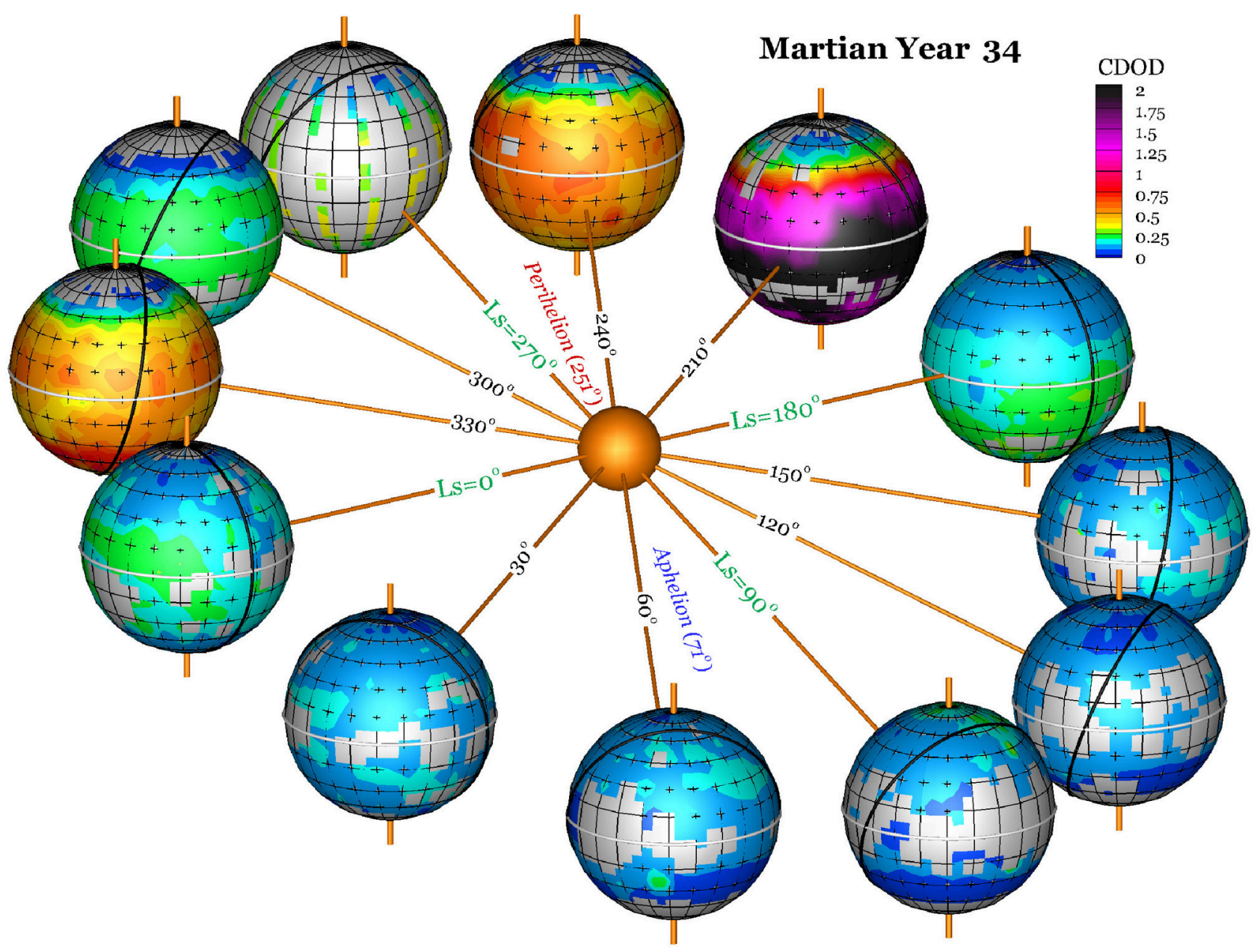

2019ja026838-f02-z-.eps

This article is protected by copyright. All rights reserved. 

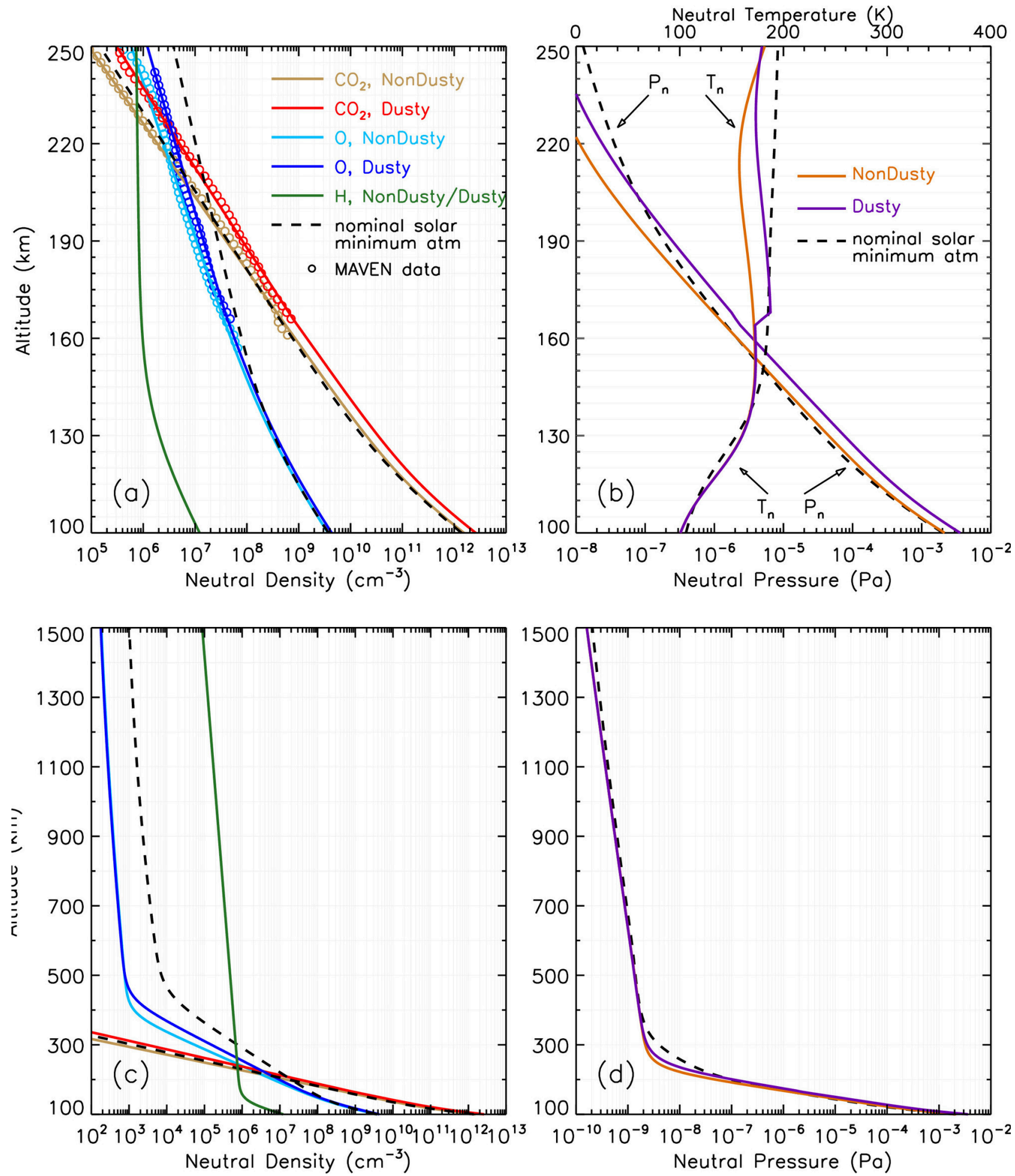

2019ja026838-f03-z-.eps 

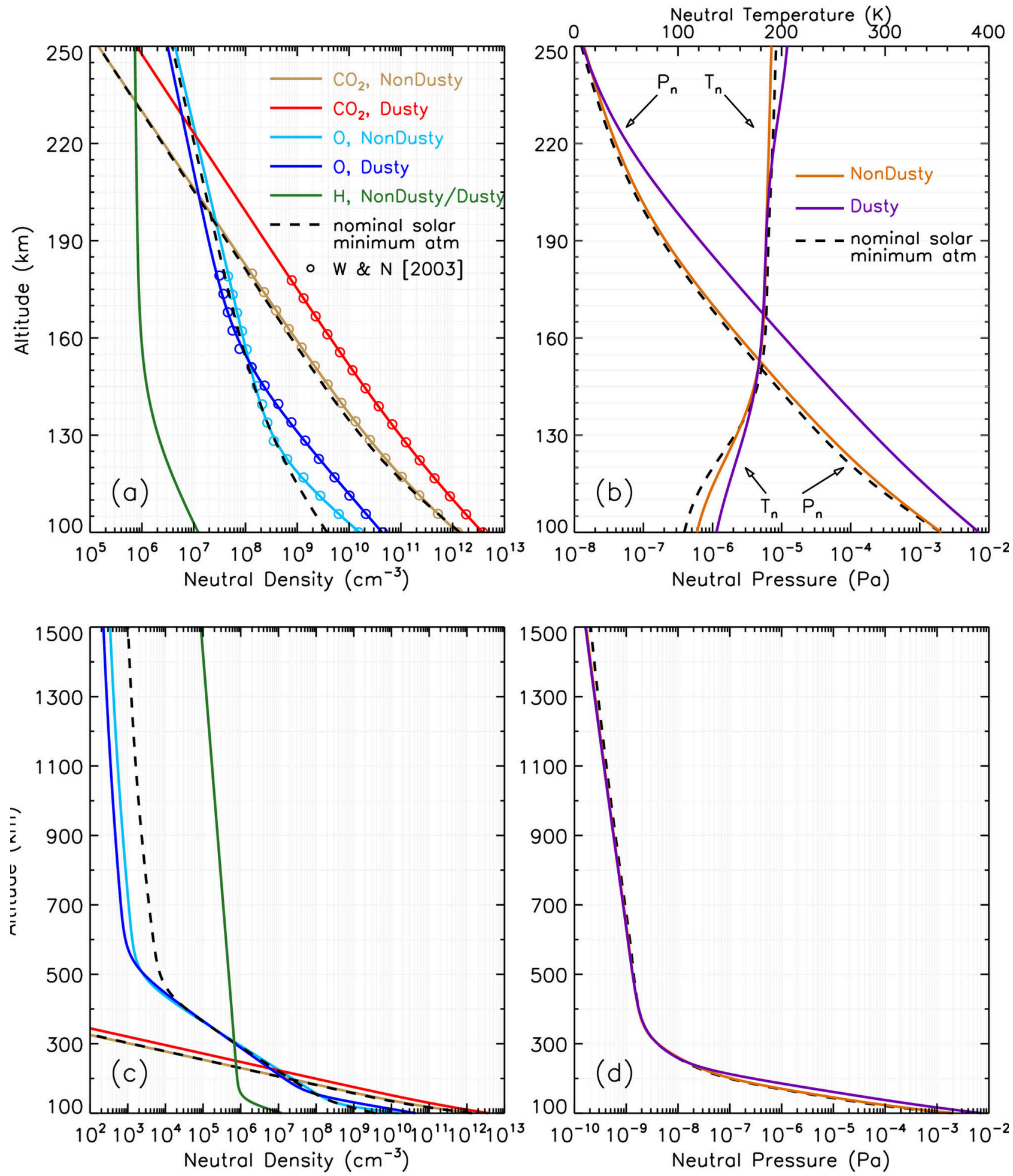

2019ja026838-f04-z-.eps 

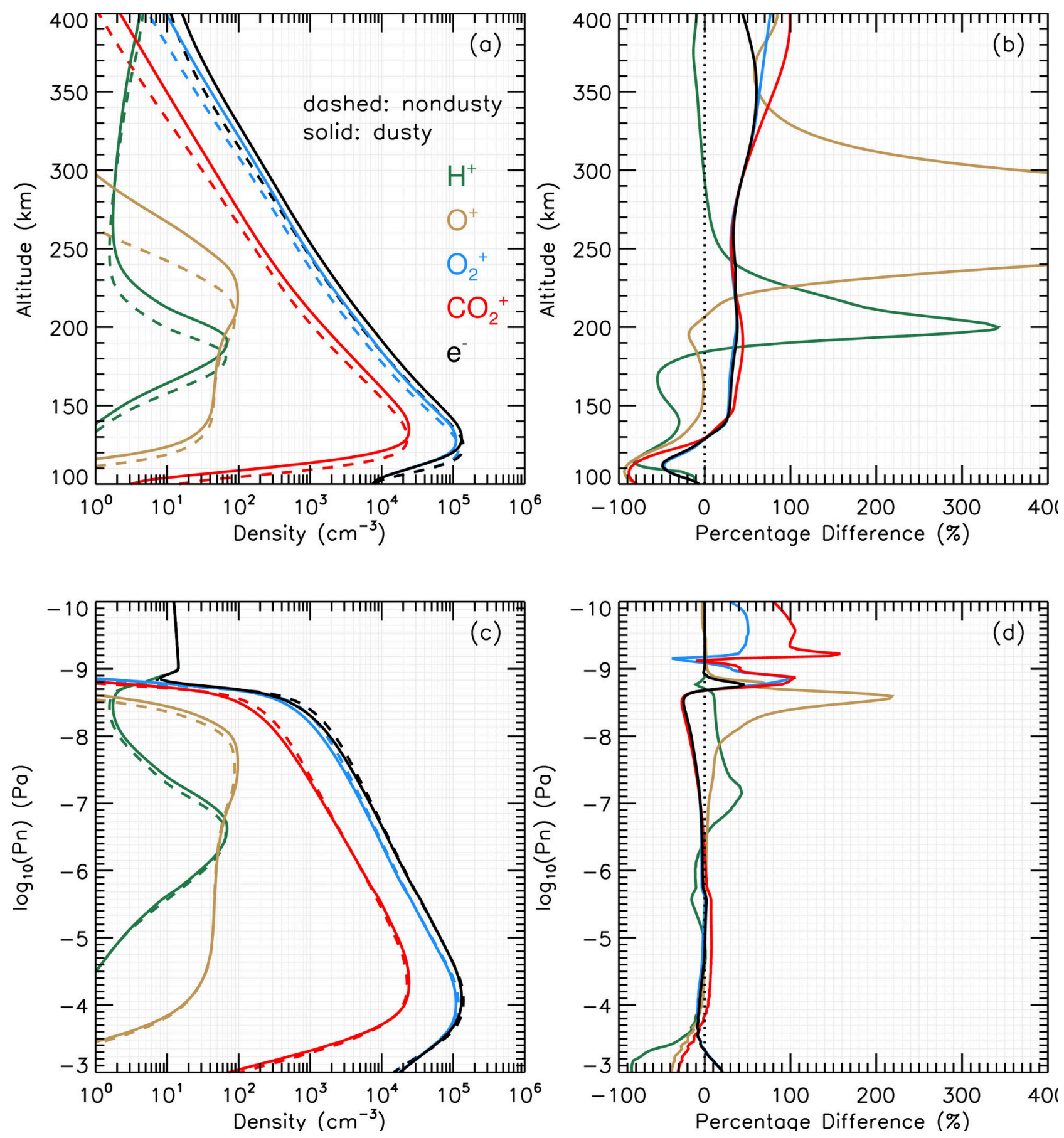

2019ja026838-f05-z-.eps 

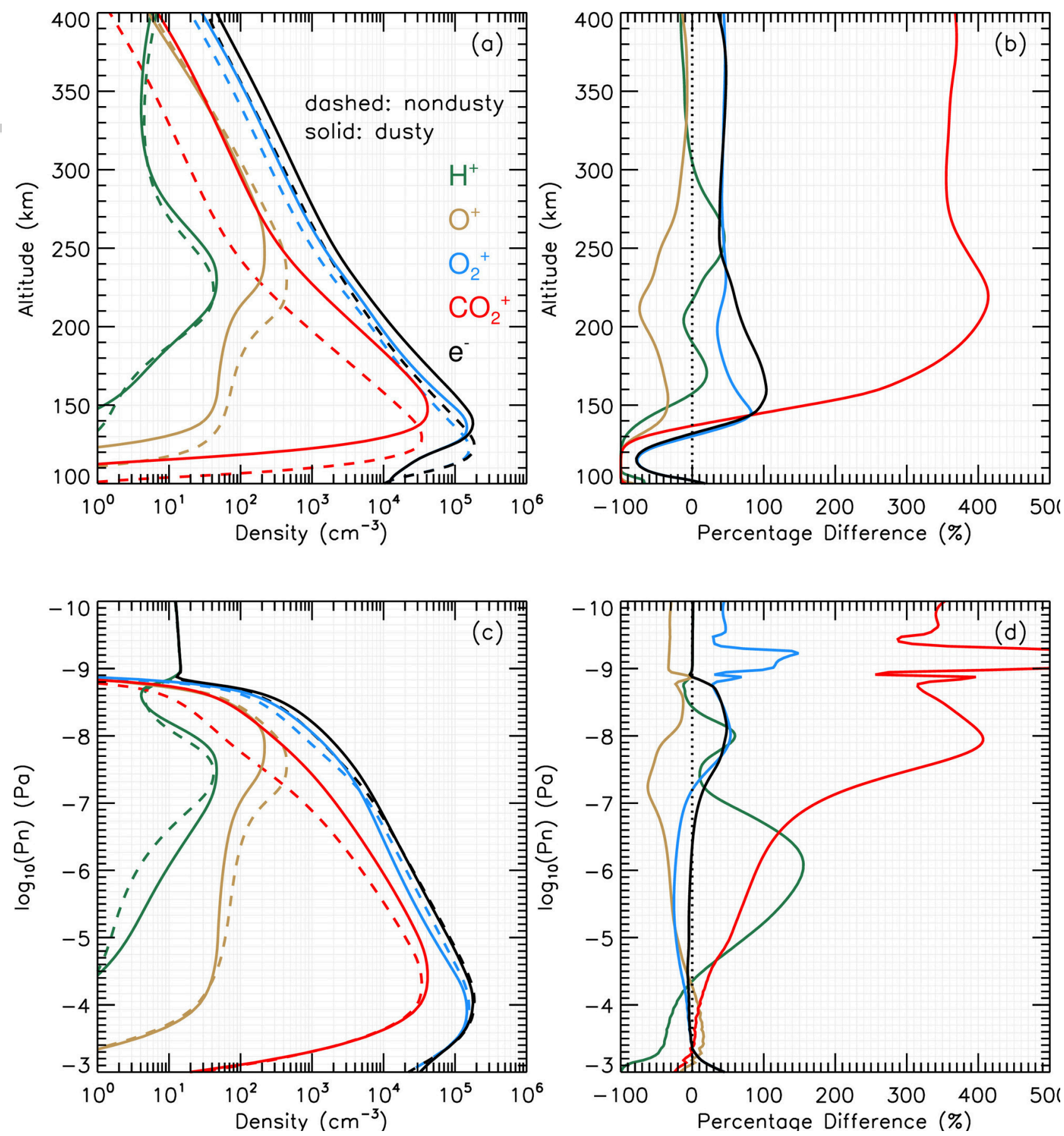

2019ja026838-f06-z-.eps 


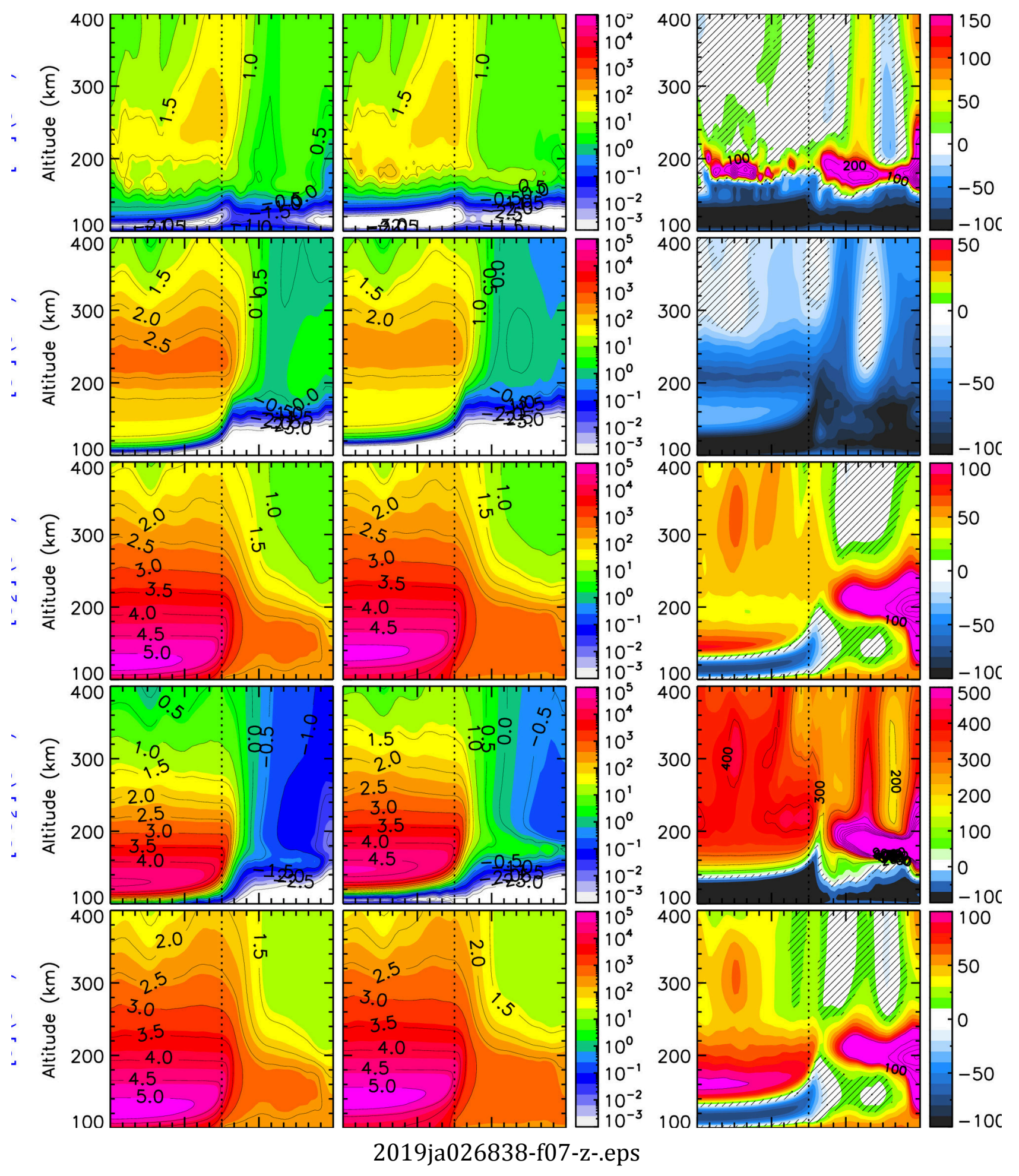

This article is protected by copyright. All rights reserved. 


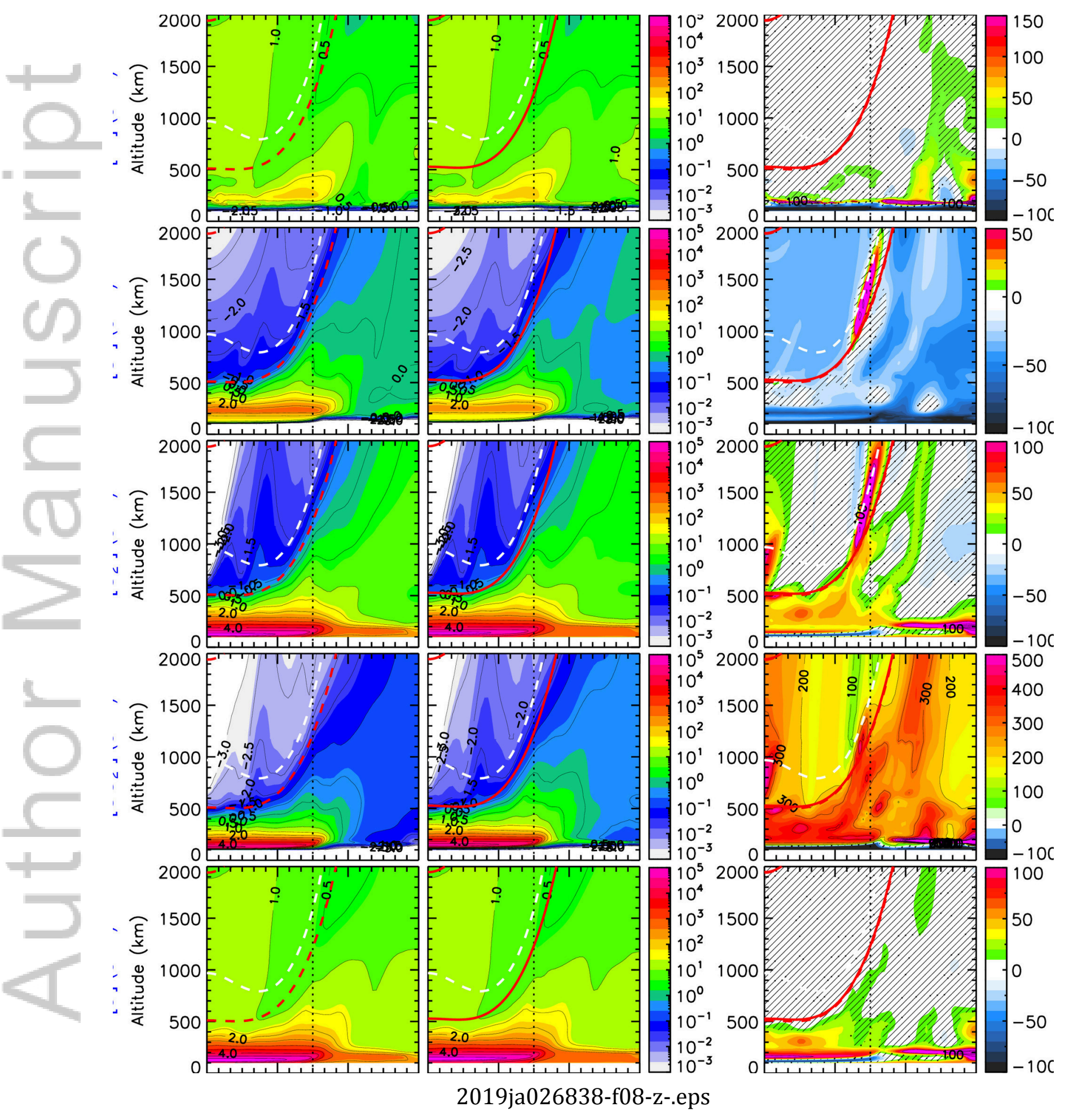

This article is protected by copyright. All rights reserved. 


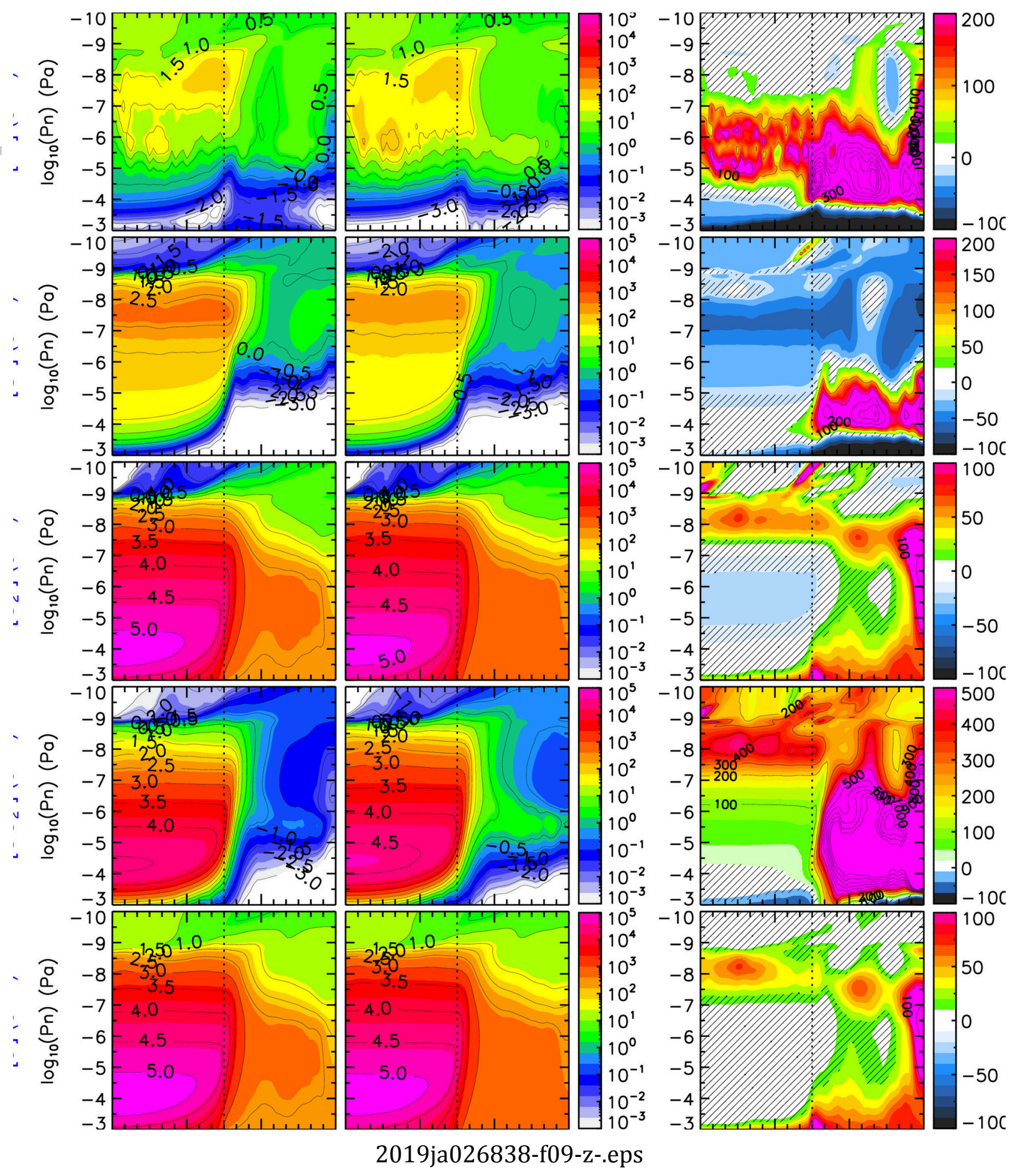

This article is protected by copyright. All rights reserved. 

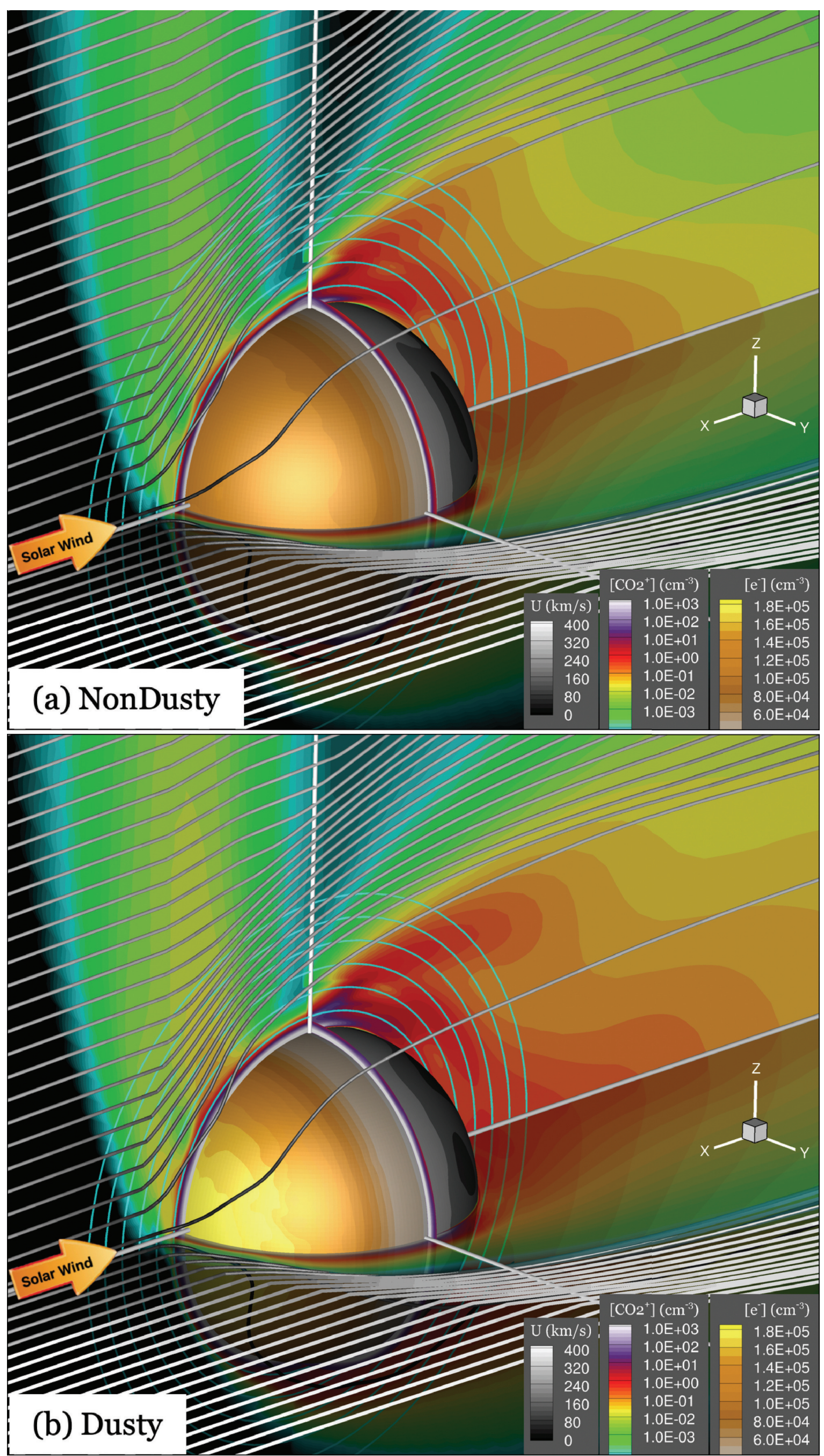

2019ja026838-f10-z-.eps

This article is protected by copyright. All rights reserved. 


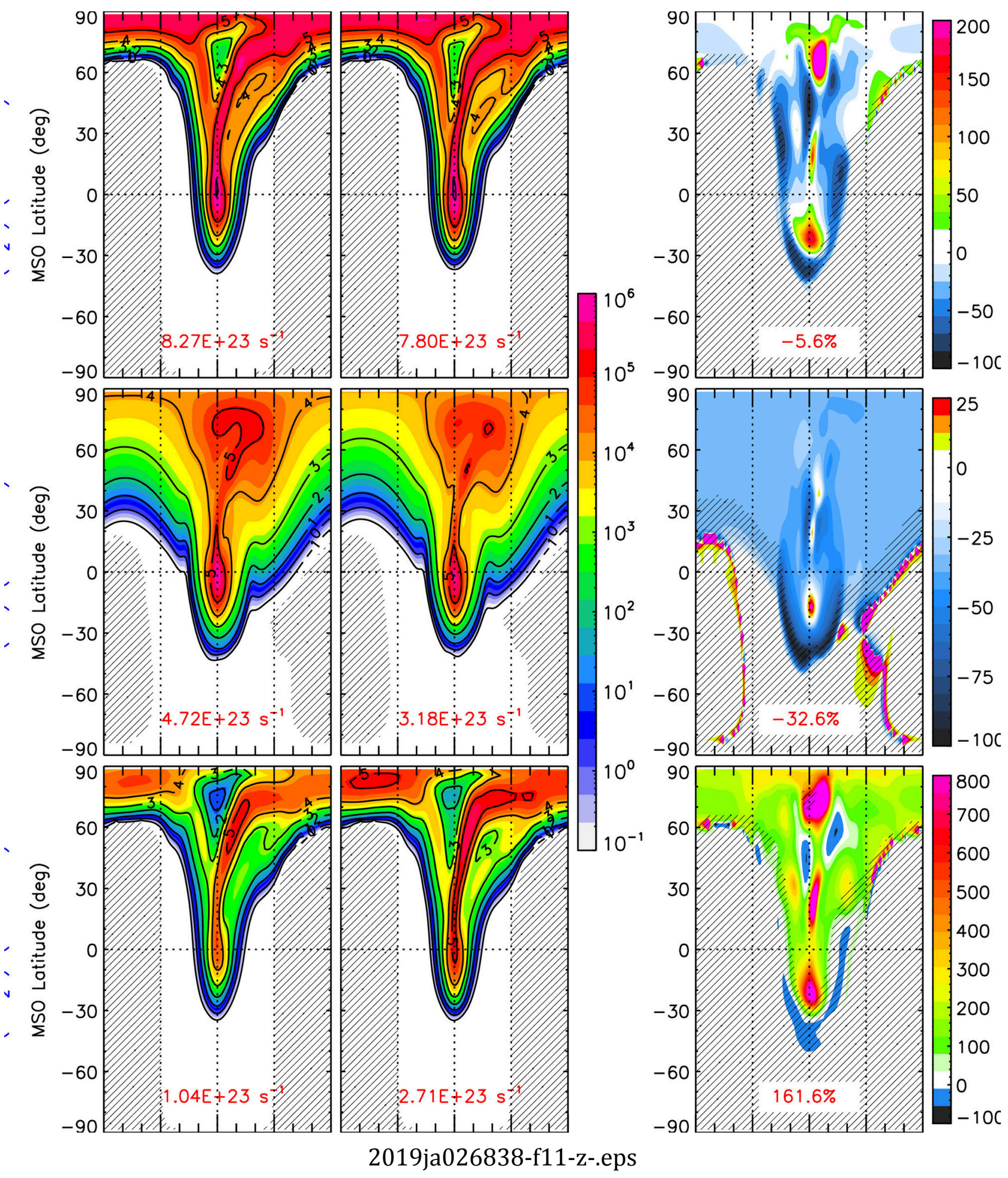

This article is protected by copyright. All rights reserved. 